San Jose State University

SJSU ScholarWorks

Master's Theses

Master's Theses and Graduate Research

Fall 2009

\title{
Relations between language and executive function in Spanish- speaking children.
}

Ana Luz Portillo

San Jose State University

Follow this and additional works at: https://scholarworks.sjsu.edu/etd_theses

\section{Recommended Citation}

Portillo, Ana Luz, "Relations between language and executive function in Spanish-speaking children."

(2009). Master's Theses. 3979.

DOI: https://doi.org/10.31979/etd.bkb9-uxfj

https://scholarworks.sjsu.edu/etd_theses/3979

This Thesis is brought to you for free and open access by the Master's Theses and Graduate Research at SJSU ScholarWorks. It has been accepted for inclusion in Master's Theses by an authorized administrator of SJSU

ScholarWorks. For more information, please contact scholarworks@sjsu.edu. 
RELATIONS BETWEEN LANGUAGE AND EXECUTIVE FUNCTION IN SPANISH-

\title{
SPEAKING CHILDREN
}

\begin{abstract}
A Thesis
Presented to

The Faculty of the Department of Communicative Disorders and Sciences

San José State University
\end{abstract}

\author{
In Partial Fulfillment \\ of the Requirements for the Degree \\ Master of Arts
}

by

Ana Luz Portillo

December 2009 
UMI Number: 1484305

All rights reserved

INFORMATION TO ALL USERS

The quality of this reproduction is dependent upon the quality of the copy submitted.

In the unlikely event that the author did not send a complete manuscript and there are missing pages, these will be noted. Also, if material had to be removed, a note will indicate the deletion.

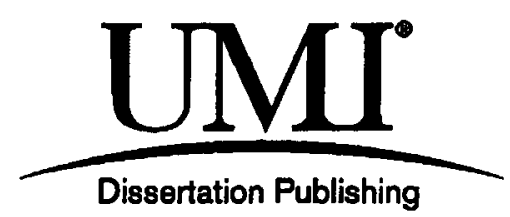

UMI 1484305

Copyright 2010 by ProQuest LLC.

All rights reserved. This edition of the work is protected against unauthorized copying under Title 17, United States Code.

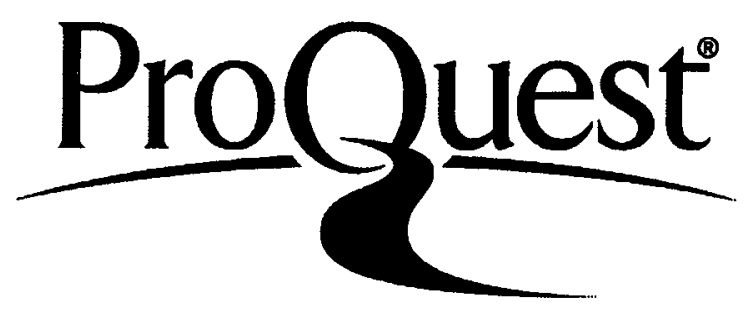

ProQuest LLC

789 East Eisenhower Parkway

P.O. Box 1346

Ann Arbor, Ml 48106-1346 
(C) 2009

Ana Luz Portillo

ALL RIGHTS RESERVED 
SAN JOSÉ STATE UNIVERSITY

The Undersigned Thesis Committee Approves the Thesis Titled RELATIONS BETWEEN LANGUAGE AND EXECUTIVE FUNCTION IN SPANISHSPEAKING CHILDREN

by
Ana Luz Portillo

APPROVED FOR THE DEPARTMENT OF COMMUNICATIVE DISORDERS AND SCIENCES

ABSTRACT

\section{RELATIONS BETWEEN LANGUAGE AND EXECUTIVE FUNCTION IN SPANISH- SPEAKING CHILDREN \\ by Ana Luz Portillo}

The literature on the development of executive function (EF) has been largely limited to studies of middle-class English-speaking children. This study extended executive function research to a primarily low socioeconomic status (SES) sample of Spanish-speaking preschool children living in the United States. The purpose of this study was to examine a variety of language measures (vocabulary, grammar, and comprehensive language) in relation to a variety of executive function measures (working memory, inhibitory control, and attention-shifting). Results revealed that Spanish speakers performed on some, but not all, EF measures comparably to higher SES English-speaking peers, in concurrence with previously reported findings. Further, language relations were strongest to the EF areas of working memory and attention-shifting. Importantly, comprehensive language measures were more powerful predictors of concurrent EF abilities than vocabulary or grammar measures alone. Theoretical and methodological implications of these findings for studies of EF-language relations in clinical populations, in children learning Spanish as a first language, and in children from low SES backgrounds are discussed. 


\section{ACKNOWLEDGEMENTS}

This thesis was possible through the collaborative efforts of dozens of individuals. Foremost, I would like to thank the thesis committee members, Dr. Jean Novak, Dr. Anne Fernald, and Dr. Michael Kimbarow, for their feedback and support from the conception of this research project nearly two years ago. I am also greatly indebted to Dr. Virginia Marchman for generously devoting time and energy to improving the manuscript to its current level.

This study would not have been possible without the parents and families who devoted their time and to the generous funding from National Institute of Health research grants to Dr. Anne Fernald. I would also like to thank all staff and students at the Center for Infant Studies. In particular, Dr. Nereyda Hurtado and Lucía Rodriguez Mata at the EPA Research Facility were wonderful, supportive colleagues, without whom I would not have so successfully completed nearly a full year of testing. The extensive data collection required for this study was greatly facilitated by their expertise and flexibility in accommodating testing sessions. Lastly, I am grateful to all my friends and family, in particular my husband Jon, for their unconditional encouragement and support, especially during the final months of writing. 


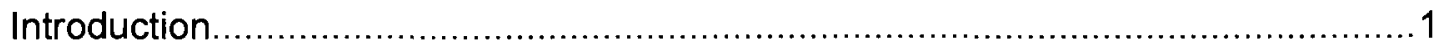

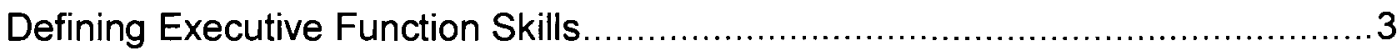

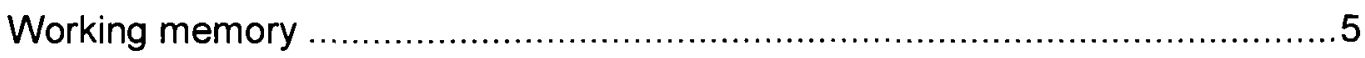

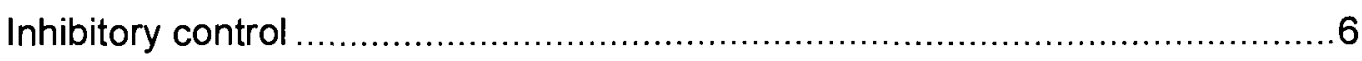

Shifting mental set, or "attention-shifting" ................................................

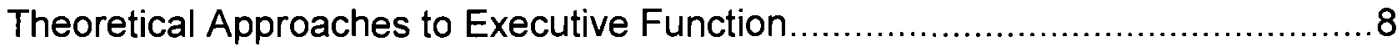

Summary of Executive Function Abilities across the Lifespan .............................

The Challenges of Measuring Executive Function Skills .................................11

Executive Function Deficits in Clinical Populations .......................................... 14

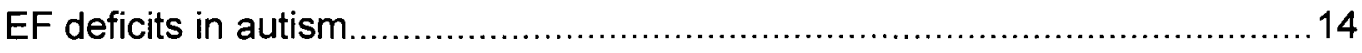

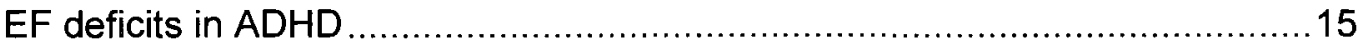

EF deficits in Specific Language Impairment (SLI) ..................................... 16

Executive Function and Language in Typically-Developing Preschoolers ..........18

Executive Function in Bilinguals and Spanish Speakers ................................20

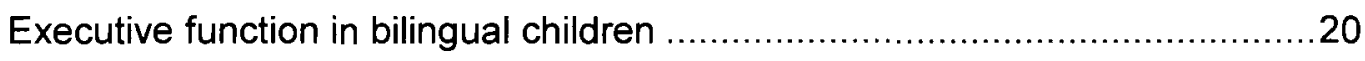

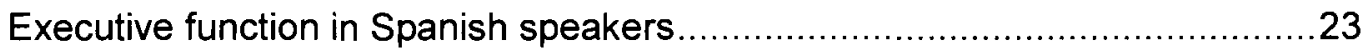

Language Assessment in Spanish Speakers ................................................25

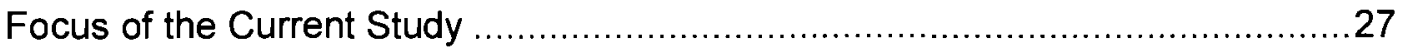

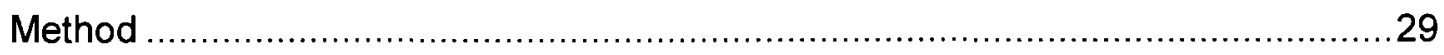

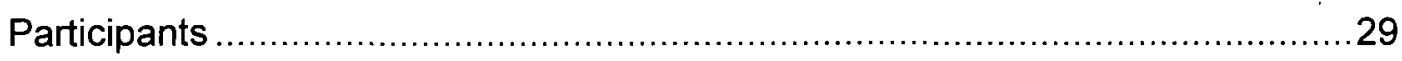

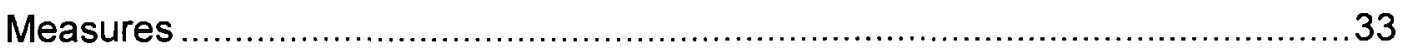

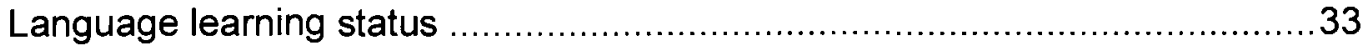



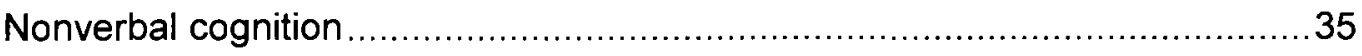

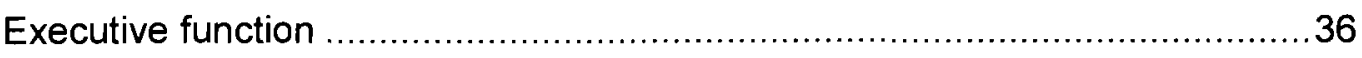

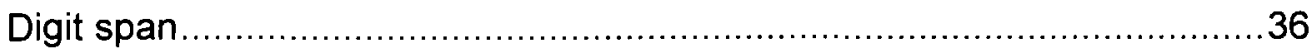

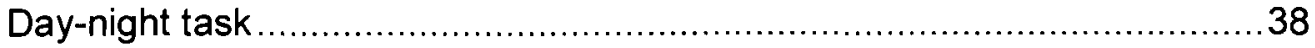

Standard Dimensional Change Card Sort (Standard DCCS) ...................... 39

Children's Behavior Questionnaire ......................................................... 41 
Language skills

Receptive One-Word Picture Vocabulary Test, Spanish-English Bilingual Edition

Spanish Preschool Language Scale, $4^{\text {th }}$ edition, expressive communication scale

Clinical Evaluation of Language Fundamentals - Preschool Edition, $2^{\text {nd }}$ edition, expressive language scale

The Bilingual English-Spanish Assessment, Spanish morphosyntax subtest 44

Reliability 45

Procedure 46

Results 47

Executive Function Measures 48

Overall performance 48

Working memory and inhibitory control 49

Attention-shifting 50

Intercorrelations among executive function measures 52

Language Measures 53

Overall performance 53

Intercorrelations among language measures 55

Relations of Language and Executive Function to SES 58

Relations between Language and Executive Function 59

Predictive Relations between Language Skills and Working Memory. 62

Relations between Language and Attention-Shifting 65

Summary of Findings 69

Discussion..... 69

Examining Executive Function Relations to SES in Spanish Speakers 70

Examining EF Measures Individually rather than as a Composite Score .74

Performance in Primarily Spanish versus Spanish-English Learners 76

Exploring Language beyond the Construct of Receptive Vocabulary 77 Limitations of the Current Study 79 


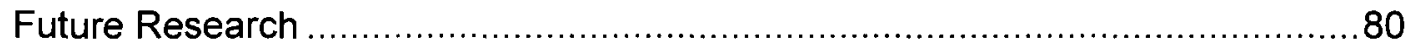

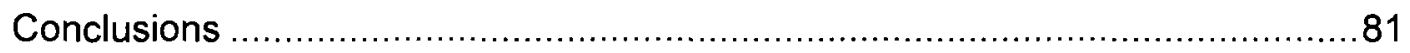

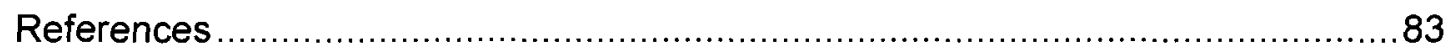




\section{List of Figures}

Figure 1. Stimulus cards used in the day-night task

Figure 2. Sorting boxes, model cards, and sorting cards used in the Standard DCCS task

Figure 3. Percentage of participants who passed the Standard DCCS task

Figure 4. Mean standard score on language assessments according to pass versus fail outcome on the Standard DCCS task for all participants $(n=36)$...66

Figures $5 \mathbf{a}$ and $\mathbf{b}$. Mean standard score on language assessments according to pass versus fail outcome on the Standard DCCS task for (a) the Primarily Spanish Learners $(n=18)$ and $(b)$ the Spanish-English Learners $(n=18)$

Figure 6. Mean composite scores on the BESA SMST according to pass versus fail outcome on the Standard DCCS task 


\section{List of Tables}

Table 1. Demographic information for all participants

Table 2. Descriptive statistics for working memory and inhibitory control measures

Table 3. Pearson correlation coefficients ( $r$ ) among working memory and inhibitory control measures 53

Table 4. Descriptive statistics for language measures

Table 5. Pearson correlation coefficients ( $r$ ) among language measures (full sample $n=36$ )

Table 6. First-order and partial correlations among language and executive function measures

Table 7. Summary of regression models using language measures as predictors of working memory performance $(n=36)$ 


\section{Introduction}

Children's acquisition of language has been of interest to researchers, as a window to underlying brain organization and development. Most children appear to develop language skills "effortlessly," through language input from people in their home and community environments. However, decades of basic language research have shown that, in fact, children use multiple and varied cues in the gradual process of language learning. These cues range from using statistical regularities to segment words in a continuous speech stream (Saffran, Aslin, \& Newport, 1997) to using social cues such as eye gaze to make inferences about the meaning of spoken words (Tomasello \& Cale Kruger, 1992). Thus, language acquisition, a task that at initial glance may appear "effortless," in fact requires fine tuning and coordination of multiple underlying cognitive processes.

While most children acquire language normally, research also shows that some children experience difficulty acquiring language compared to same-age peers. This difficulty can stem from broader underlying cognitive deficits, as seen in mental retardation or Down syndrome. However, a language disorder can also occur in the absence of any other cognitive or developmental delay, as in the case of Specific Language Impairment (SLI; Leonard, 1998). Understanding disorders in which language fails to develop normally helps in turn to inform the study of normal language development. Although it is clear that a deeper understanding of language development and disorders is beneficial, studies of language and disorders have been limited in several ways. One important limitation is that studies have primarily focused on middle class English speakers. Thus, one purpose of this study is to explore language skills in Spanish-speaking children. 
An important theoretical issue arises from research on children with normal language development and those with language disorders. How are language skills in typical and atypical development related to other cognitive skills? To shed light on this question, the current study focuses specifically on the set of cognitive skills referred to as executive function. Executive function (EF) is an umbrella term referring to higherorder skills that allow people to carry out goal-directed behavior in novel problem-solving situations (Anderson, Anderson, Jacobs, \& Smith, 2008; Banich, 2009; Garon, Bryson, \& Smith, 2008; Jurado \& Rosselli, 2007). Some of these skills include setting goals, planning step-by-step solutions to problems, updating information in working memory, maintaining focus on the task at hand in the presence of distracters, and inhibiting inappropriate responses (Anderson et al., 2008; Banich, 2009; Garon et al., 2008; Jurado \& Rosselli, 2007).

Although the importance of EF research is well-established, there is a bias in the executive function literature focusing on EF skills of middle-class English speakers. Expanding EF research to Spanish is particularly relevant given that Spanish is currently the biggest minority language group in the United States (US Census, 2000, www.census.gov). Data from the 2000 US Census indicates that Spanish-speaking children comprise over three-quarters of English-language learners (ELLs) in the United States. In addition, Latino children in the United States are more likely to come from families of lower socio-economic status (SES) backgrounds compared to their nonLatino white peers (Brindis, Driscoll, Biggs, \& Valderrama, 2002), and hence, may be at increased risk for later academic problems compared to higher-SES peers. A deeper understanding of language and EF development in Spanish speakers is therefore critical for the development of effective and efficient ways to serve this growing population. 
This study explores relations between language abilities and EF performance in predominantly Spanish-speaking preschoolers living in the United States. Participants in this study were part of a larger longitudinal study of Spanish language development. Although all children began acquiring Spanish as their first language (L1), over time some participants had been exposed to English. Therefore, in addition to investigating EF skills in Spanish speakers, the current research may also contribute to the understanding of EF skills in children in early stages of second-language learning.

Before turning to the main research questions in this study, several literatures are reviewed. First, definitions of executive function are presented, along with prominent theoretical approaches in the study of EF and the development of EF components from infancy through adulthood. Three EF areas in particular are examined in the current study: working memory, inhibitory control, and attention-shifting. A review of EF and language relations in typically-developing preschoolers is presented, followed by a review of EF in special populations. Lastly, the EF literature on Spanish speakers and second-language learners is presented, and the main goals of the current research are outlined in greater detail.

\section{Defining Executive Function Skills}

Traditionally, researchers have viewed executive function skills as being housed in the frontal lobe of the brain. From an evolutionary standpoint, the frontal lobes are the youngest part of the human brain and are also the largest in humans compared to other animals. In addition, compared to other brain areas, the frontal lobes have the slowest course of maturation, extending through roughly 25 years of age (Sohlberg \& Mateer, 2001). The frontal lobe also appears most vulnerable to effects of aging, with 
disproportionately higher rates of cell shrinkage and volume loss (Jurado \& Rosselli, 2007).

Executive function encompasses a wide array of different skills necessary for everyday problem-solving. Anderson, Anderson, Jacobs, and Smith (2008) describe EF as occupying "a managerial role" (p.124) in information processing and behavior. Jurado and Rosselli (2007) write: 'in a constantly changing environment, executive abilities allow people to shift their mind set quickly and adapt to diverse situations while at the same time inhibiting inappropriate behaviors" (p.214). Some specific EF skills cited in the research literature include: goal setting and monitoring, creativity in generating solutions to novel problems, planning, initiation, self-regulation and impulse control, working memory, and cognitive flexibility, especially upon encountering barriers to attainment of goals (Anderson et al., 2008; Banich, 2009; Garon et al., 2008; Jurado \& Rosselli, 2007).

There is also a distinction between "cold" and "hot" executive function areas (Anderson et al., 2008; Garon et al., 2008). The term "cold" refers to more purely cognitive EF areas that are affect-neutral, such as working memory skill, whereas "hot" refers to affect-based or reward-sensitive areas. For instance, in reward delay tasks children may be told that they can eat a chocolate chip cookie whenever they choose simply by ringing a bell. However, if they refrain from ringing the bell and instead wait for an adult to return to the room after a brief delay, they receive double the amount of chocolate chip cookies (Carlson, 2005). Such "hot" EF tasks are viewed as activating different pleasure and reward centers of the brain compared to "cold" EF tasks.

Although the list of EF areas is lengthy, there is ongoing debate among different researchers about which subcomponents should be included in a formal definition, and 
which should be viewed as related to, but not core components of EF (Anderson et al., 2008). Thus, there currently is no universally agreed-upon definition of executive function. In the current study, three EF areas were chosen, each of which is further detailed below.

Working memory. Working memory refers to the ability to hold information in mind in order to complete a task (e.g., remembering a verbal direction in order to follow it). Several models of working memory have been presented in the research literature, and two of them will be referenced in this paper. Arguably the most influential model in the literature is that of Baddeley (1996). He views working memory as a three-part structure, run by an attentional control system (termed the "central executive") that allows a person to focus and switch attention among tasks. Baddeley's model posits that there is sensory-specific storage. The "phonological loop" is for maintaining shortterm auditory information, and is thus involved in language processing, while the "visuospatial sketchpad" processes information from the visual domain. The most critical element of this model as it relates to EF is that the "central executive" is ultimately responsible for allocating attention resources to different cognitive processes.

In contrast, Just and Carpenter (1992) describe a limited-capacity model that includes a more general set of resources for supporting language processing. They propose that activation is what impacts both information processing abilities and storage capacity. Individual differences in amounts of activation available in working memory translate into different working memory capacity for language processing among individuals. In their model there is a tradeoff between capacity and processing. When activation demands exceed storage capacity in working memory, language processing 
suffers. Conversely, when complex linguistic processing is required (e.g., in cases of syntactic ambiguity), a reduction in storage capacity is evident.

Inhibitory control. The second EF area of focus in the current study is inhibitory control, also known as response inhibition. Inhibitory control refers to the ability to regulate automatic response tendencies. For example, inhibitory control may involve a kindergartener controlling the urge (i.e. automatic response tendency) to blurt out an answer in class. In this example, a child with inhibitory control would raise his hand and wait to be called upon by the teacher. The popular children's game "Simon Says" is another example of inhibitory control. In this game, children must only act out actions that are preceded by the carrier phrase "Simon says..." This is difficult given that the automatic response tendency for young children is to act out a verbal command.

In more structured laboratory-based tasks, inhibitory control is manifested both at cognitive levels and at behavioral levels (Marton, Kelmenson, \& Pinkhasova, 2007). A "cold" EF task tapping into inhibitory control at cognitive levels might involve resisting the urge to respond to certain stimuli. For example, the computerized "Go No-Go" task is often used as an inhibitory control measure (Logan \& Cowan, 1984). In this task, participants must press a key on the keyboard for all letters that appear on the screen, with the exception of one target letter, such as R. Given that the automatic response is to press a key for all letters, inhibitory control is required to refrain from pressing a key when shown the target letter $\mathrm{R}$.

The most well-known and frequently used task to assess inhibitory control in adults is the Stroop Color and Word Test (Golden, 2002). In this task, participants are shown several words corresponding to color names (e.g., red, green, blue). However, each word is written in color different from the color indicated by the word. For example, 
the word "red" may be written in a blue color. To complete this task, adults must inhibit reading the word (an automatic response tendency for adults) and instead say the color in which each word is written. While this task is appropriate for measuring inhibitory control in adults, it cannot be used for young children given the need for adult-level reading skills. However, Gerstadt, Hong, and Diamond (1994) developed a Stroop-like EF equivalent for use with children. Their task, called the day-night task, involves responding "day" to a card with a picture of the moon and "night" to a card with a picture of the sun. The automatic response to give the corresponding label for the picture must be inhibited, and instead the opposite response must be given.

Shifting mental set, or "attention-shifting." The final EF area of interest in the current study is shifting mental set, also referred to as attention-shifting. Attention involves being able to focus on a task over time (focused attention), in the presence of competing tasks or distractions (selective attention), as well as being able to shift focus from one task to another (alternating attention; Sohlberg \& Mateer, 2001). In the EF literature, attention-shifting first requires that attention be directed to one response rule. Then, the response rule changes in such a way that the new response set is in conflict with the old response set.

One classic measure of attention-shifting in the neuropsychology literature is the Wisconsin Card Sorting Test (Grant \& Berg, 1993). In this test, individuals are shown stimulus cards that vary on three dimensions: shape (e.g., triangles, circles, squares), color (e.g., blue, red, green), and quantity (one, two, or three objects). Three model cards are initially laid out, each of them different from the others in all dimensions. For instance the first model card might contain one blue triangle, the second card two red circles, and the third card three green squares. The experimenter picks a target 
dimension (e.g., color) but does not inform the participant of his choice. The participant is simply handed a stack of stimulus card and told to begin sorting them in different piles. The experimenter provides verbal feedback ("right" or "wrong") to the participant after each stimulus card is sorted, and the participant has to deduce the sorting rule based on the feedback alone. At some point after correct sorting is achieved on the first target dimension (color, in this example), the experimenter switches to a new sorting rule (e.g., number), also without informing the participant. Attention-shifting ability is assessed by looking at patterns of errors committed after the new sorting rule was in place.

Perseverative errors in sorting according to the first target dimension (color, in this example) would be indicative of failure to shift mental set.

Theoretical Approaches to Executive Function

Several theoretical approaches to understanding EF have emerged in the research literature. Among the first to emerge was the view that executive function is a unitary construct (Jurado \& Rosselli, 2007). Proponents of this view believe that there is a central ability that underlies goal-directed behavior. Salthouse (1996) hypothesizes that general information processing speed, in particular, may be the skill underlying executive function performance, while other researchers speak more broadly of "general intelligence" as the underlying ability affecting all EF areas (Obonsawin et al., 2002).

A more recent framework for conceptualizing EF has been the view that EF is comprised of dissociable components (Diamond, 2006) that interact with each other to coordinate behavior. Working memory and inhibition are among the main dissociable components in this view. The presence of distinct developmental trajectories and different growth rates among the subcomponents has been used to support this view of EF (Carlson, 2005; Diamond, 2006; Garon et al., 2008). Other support stems from low 
intercorrelations among tasks measuring executive function skills (Jurado \& Rosselli, 2007).

Most recently, some researchers have conceptualized EF using an "integrative framework" where EF is viewed as both a unitary construct but also is believed to have dissociable components (Miyake et al., 2000). More specifically, Miyake et al. says that while studies point to some clearly dissociable components, there are some abilities underlying several EF skills. One possibility for this "underlying ability" may be a basic inhibitory control mechanism that requires, for instance, the ability to ignore information as it becomes irrelevant through various stages of goal attainment (Miyake et al., 2000). Another possibility is a general working memory ability that allows people to update goals within changing environmental contexts.

\section{Summary of Executive Function Abilities across the Lifespan}

The development of executive function abilities across the lifespan has been well-documented, albeit better in some areas of EF than others. Garon, Bryson, and Smith (2008) argue that the development of attention is perhaps the most important precursor to the emergence of other EF skills. First, infants acquire the ability to focus on a single stimulus over time. Selective attention is a skill that progresses well into childhood but shows particular growth during the preschool years. Evidence of EF-like, goal-directed behavior is also manifested in infancy. For example, older infants persist in reaching toward an object of interest until they succeed at obtaining it. In addition, working memory abilities have been documented in children as young as 6 months of age through the use of delayed response tasks, where infants must hold object representations in mind over a delay (Diamond, 1985). 
The preschool years have been documented as a time of rapid growth in several EF areas. This is one reason the preschool age-group was selected for this study. At this age, working memory is often assessed through span tasks, where children must repeat increasingly longer lists of words or numbers. Such tasks have yielded individual differences in preschool-aged children. Set shifting, also known as cognitive flexibility, or the ability to change a response set, emerges between 3 and 5 years of age and is assessed primarily through switch tasks. In switch tasks, children are asked to sort stimuli by one dimension, such as color. Then a new rule is introduced (e.g., to sort stimuli by another dimension, such as shape), and 3-year-old children are typically unable to "switch" to sorting by the new dimension (Zelazo, Frye, \& Rapus, 1996).

EF development does not end in early school-age, but rather is as a "protracted process" (Anderson et al., 2008; p.123) that extends at least through adolescence. Verbal fluency, among the last EF skills to surface, refers to the ability to generate category members under a time constraint (Jurado \& Rosselli, 2007). This skill is typically measured through use of phonemic or semantic fluency tasks. In phonemic fluency tasks, individuals are asked to name as many words as possible in one minute that begin with a certain letter, such as " $F$." In semantic fluency tasks, individuals may be asked to name as many fruits as possible within a specific time interval. Verbal fluency ability is shown to emerge around age 6 and to develop roughly through 15 years of age (Jurado \& Rosselli, 2007). This EF skill involves both the ability to generate novel responses according to some category, as well as to inhibit responses that do not satisfy the target category.

Finally, deterioration of EF skills in aging has been well documented and is linked to significant reductions in size and volume of the frontal lobes during the normal aging 
process. Among the most prominent areas affected are inhibition of irrelevant information when problem-solving, short-term memory, set-shifting, and category fluency (Jurado \& Rosselli, 2007). This decline in EF skills during aging has led researchers to propose that several executive function sub-areas follow a U-shaped developmental pattern: poor performance in childhood, peak performance in young adulthood, and a return to poor performance in old age (Zelazo, Craik, \& Booth, 2004).

In summary, the task of defining executive function is challenging given that there are different theoretical frameworks for studying EF. There is continued disagreement about which skills fall under the EF umbrella and which are related to, but not core components of executive function. Proponents of the unitary view conceptualize EF as being driven by a central underlying ability, whereas the dissociable components approach views EF as composed of distinct skills that interact with each other in processing. Finally, some authors advocate an integrative framework where EF has distinct components but is also mediated by an underlying ability. Studies tracking the development of EF skills show that many EF areas follow a U-shaped developmental trajectory, where skills are gradually strengthened through adulthood and are weakened again in old age. In the next section, general methodological challenges in measuring EF are presented.

\section{The Challenges of Measuring Executive Function Skills}

Historically, executive function tests were purported to measure abilities housed in the frontal lobe. As such, the psychometric sensitivity of most clinical tests is at the level of identifying frontal lobe damaged patients from non-damaged controls (Jurado \& Rosselli, 2007). While in some clinical cases this level of sensitivity may be helpful in identifying patients with EF deficits severe enough to prevent independent living, such 
tests are not sufficient for examining individual differences in EF abilities in neurologically intact individuals. Use of functional neuroimaging in EF emerged, in part, from this need for finer-grained, objective measurements. Using this method, imaging data is collected during tasks thought to tap into executive function. However, interpreting the results is complicated at best, given that most tasks require many EF skills (e.g., attention, working memory, sequencing, planning). Therefore, obtaining "pure" measures of individual EF components is difficult, and ways to isolate contributions of individual components, such as attention from working memory are still being explored (Banich, 2009).

Use of norm-referenced, standardized tests is another option for assessment of EF ability. However, researchers discuss the difficulty of developing standardized tests of executive function abilities because, by definition, executive function involves problem-solving behaviors in response to novel stimuli (Banich, 2009; Jurado \& Rosselli, 2007). Executive function skills emerge in "novel, unstructured and non-routine situations that require some degree of judgment" (Banich, 2009, p.89). However, standardized tests are highly-structured, with the test administrator in charge of providing a controlled environment that is free of distracters. Additionally, establishing psychometric properties, such as test-retest reliability, is difficult given that stimuli lose novelty during a repeated test administration (Jurado \& Rosselli, 2007). Banich (2009) also raises the issue that there is no executive function "gold standard" relevant to measuring EF in the laboratory, especially through the use of standardized tests.

In addition to developing tasks that accurately capture EF skills, there is the additional challenging of obtaining adequate measures of EF skills in children, who are a moving target when it comes to EF skills. Perhaps the greatest challenge to measuring 
EF abilities in young children is the limited availability of developmentally appropriate assessment tools (Anderson, 2008). This is likely due to children's limited language ability compared to fully-developed adults (Jurado \& Rosselli, 2007). In addition, it is also difficult to know how well the critical executive function component has been preserved when modifying an adult executive function task for use with young children (Garon et al., 2008).

A third issue surrounding EF measurement in children is knowing the age range for which a particular EF task will capture variability in performance. In an effort to address this issue, Carlson (2005) collected data from 600 typically-developing preschoolers in order to document age-related changes from 2 to 6 years in over 20 different executive functioning tasks. She provided statistical analyses for the probability of passing each EF task as a function of the child's age. For example, at 2 years of age, children have approximately a $50 \%$ probability of passing a snack delay task. The level of detail and analysis provided in Carlson's review of preschool EF tasks is invaluable to researchers investigating EF at the preschool age.

In the current study working memory is assessed using a verbal digit span task requiring children to repeat sequences of numbers. Inhibitory control is measured through the day-night task and through parent-report of inhibitory control, the Children's Behavior Questionnaire (CBQ). Use of the CBQ has been well-established and documented in the literature (Putnam \& Rothbart, 2006; Rothbart, Ahadi, Hershey, \& Fisher, 2001) with both English- and Spanish-speaking children. Lastly, the area of attention-shifting is measured using the Standard DCCS task. It should be noted that while this is the most frequently-used measure of attention-shifting at the preschool age, there are no studies examining performance on the Standard DCCS task in Spanish- 
speaking children. The vast majority of studies have tested English-speaking American children from middle class backgrounds, and even the one study that used the Standard DCCS task with Chinese-English bilingual children administered it in English to all participants (Bialystok, 1999). Therefore, the current study will be the first to present data on the Standard DCCS task in Spanish-speaking children.

Intercorrelations among the executive function tasks will reveal to what degree each task measured different subcomponents of the executive function umbrella.

Significant correlations among EF measures would suggest overlap in EF areas, while non-significant correlations among EF measures would indicate that the three EF tasks administered to children sampled unique, non-overlapping EF skills.

\section{Executive Function Deficits in Clinical Populations}

Various degrees of executive function (EF) impairment have been documented in clinical populations, including brain injury, developmental disorders such as autism spectrum disorder and attention deficit hyperactivity disorder (ADHD), as well as specific language impairment (SLI). The research findings surrounding EF in autism, ADHD, and SLI are briefly summarized below.

EF deficits in autism. Executive function deficits in children with autism are well-documented but have not been found consistently across different EF areas or for different age groups. One possibility for this inconsistency is that autism includes children with a wide range of abilities. Some children with low-functioning autism are completely nonverbal whereas other children with high-functioning autism may perform with normal limits on tests of language but adequate social communication skills. Evidence of executive function deficits in school-age children with autism has been welldocumented. Even when controlling for age, verbal and nonverbal IQ, school-age 
children with autism perform significantly lower than typically-developing peers on tasks involving complex working memory and a combination of working memory and inhibitory control, (Joseph, McGrath, \& Tager-Flusberg, 2005).

While executive function deficits in school-age children are robust, EF deficits in preschool-aged children with autism have not been found consistently across different studies. One possibility for this is that executive function deficits may surface around the preschool-age in children with autism. In other words, EF impairment may be a secondary deficit of the disorder (Yerys, Hepburn, Pennington, \& Rogers, 2007). Dawson, Meltzoff, Osterling, and Rinaldi (1998) found 5-year-olds with autism performed significantly worse on measures of working memory, abstract rule learning, and response inhibition, compared to both typically-developing peers and to children with Down Syndrome (matched for chronological age and nonverbal IQ). Griffith, Pennington, Wehner, and Rogers (1999), however, found no differences in working memory, response inhibition, and attention-shifting in children with autism ages $3 ; 4-5 ; 1^{1}$ compared to a control group of children with a variety of other developmental delays. However, children with autism did have significantly fewer joint attention and social interaction events as measured by the Early Social Communication Scales (Mundy et al., 2003), a structured observation measure of nonverbal communication skills in children $0 ; 8-2 ; 6$. Taken together, this evidence suggests that the preschool age may be a critical time for capturing EF deficits. However, further research is necessary for understanding for certain whether EF deficits in fact surface around this age.

EF deficits in ADHD. According to diagnostic criteria, children with ADHD have deficits in attention, impulsivity, and sometimes in hyperactivity (American Psychiatric Association, 2000), all of which are implicated in executive control. Interestingly, 
children with ADHD are qualifying for speech and language intervention services at increasing rates. For example, Oram, Fine, Okamoto, and Tannock (1999) found that children ages $7 ; 0-11 ; 0$ with ADHD alone or with co-morbid ADHD and language impairment scored significantly worse than normal peers on an expressive language subtest that involves the executive function areas of attention, working memory, inhibition, and organization. In contrast, language subtests that were less taxing (either they relied mostly on working memory or did not require much organization and inhibition) were not more difficult for ADHD children compared to typically-developing peers. These findings are just one example of how executive function impairment can be detrimental to expressive language performance.

EF deficits in Specific Language Impairment (SLI). Children with SLI have impaired language in the absence of other cognitive impairments, including nonverbal IQ (Leonard, 1998). Unlike other populations of children with language disorders (e.g., children with autism, ADHD, or other developmental delays), SLI appears to uniquely affect language ability. Given this "pure" disorder of language, extensive research has been conducted with this population. In the area of executive function, working memory deficits have been reliably documented (Dollaghan \& Campbell, 1998; Ellis Wesimer, Evans, \& Hesketh, 1999; Montgomery, 1995; Montgomery, 2003). Some researchers believe that children with SLI show "generalized slowing" across linguistic and nonlinguistic tasks alike (Bavin, Wilson, Maruff, \& Sleeman, 2005). Others, however have found intact nonverbal working memory abilities (Riccio, Cash, \& Cohen, 2007) and used this as evidence that working memory deficits in SLI are specific to processing, manipulation, and storage of information from the verbal domain. The literature on working memory abilities in children with SLI has also given some attention to possible 
links between working memory and inhibitory control. For example, Marton, Kelmenson, and Pinkhasova (2007) found that children with SLI committed more immediate inhibitory control errors and perseverative errors on a working memory listening span task compared to age-matched and language-matched peers.

Deficits in specific types of attention in SLI are documented for certain modalities. Spaulding, Plante, and Vance (2008) found that children with SLI do equally well compared to TD peers on attention orienting (i.e. responding to stimuli) but they may be impaired on sustained or divided attention. Also, their attention skills may differ depending on the sensory modality (visual, nonverbal-auditory, or linguistic) and on degree of attentional load (low versus high). High attentional load conditions include background noise and more closely mimic everyday environments where children must use attentional resources to guide their focus. In their study, performance in the visual domain was equal for both TD and SLI groups in both low and high attentional-load conditions, but a different pattern of results emerged on nonverbal-auditory and linguistic stimuli. SLI children performed significantly worse in the high attentional load condition on both nonverbal-auditory and linguistic stimuli. Performance was equal for both groups in the low attentional load condition. These findings provide evidence that selective attention capacity is not diminished across all sensory domains, but may be specific to the auditory domain under increased conditions that require increased attention.

There are no known studies examining the shifting of mental set, or attentionshifting abilities in children with SLI. One possible explanation for this is that measures of attention-shifting use linguistically-loaded tasks, such as the Standard DCCS task where children must carefully listen to the experimenter's verbal directions in order to 
complete the task. Such a task would confound measures of attention-shifting with language ability. Other attention shifting tasks such as the Wisconsin Card Sorting Test do not require heavy language processing. However, they may be beyond a child's developmental level, or may tax working memory, which is also known to be impaired in SLI. The development of attention-shifting measures with relatively low linguistic and working memory demands would be ideal for assessing ability in this EF area, but such measures have yet to be developed.

\section{Executive Function and Language in Typically-Developing Preschoolers}

Studies focusing on the preschool age group examine EF areas of working memory, inhibitory control, and attention. Wolfe and Bell (2004) tested 41/2-year-old English-speaking children using the Peabody Picture Vocabulary Test, $3^{\text {rd }}$ ed. (PPVT-III; Dunn \& Dunn, 1997), a standardized test of receptive vocabulary, and two inhibitory control tasks, the day-night and the yes-no tasks. In the yes-no task, children were instructed to say "yes" when the experimenter shook her head no, and "no" when the experimenter nodded her headed yes. The researchers computed a composite inhibitory control score (average of percent correct on the two tasks) and used a median split to group children into either high or low inhibitory control groups. They found that children in the high inhibitory control group had significantly higher PPVT-III scores compared to peers with low inhibitory control.

In a later study investigating age-related differences in working memory and inhibitory control (WMIC) and their relation to language, Wolfe and Bell (2007) broadened the EF measures to five tasks sampling working memory and inhibitory control abilities. First, a "goldfish" task was administered to test children's ability to inhibit eating a goldfish cracker placed on the tip of the tongue over increasing time 
intervals from 10-30 seconds. Children also completed a Simon-says-like task where two puppets gave the children actions to perform, but children were instructed to perform the actions given by one of the puppets only. The third and fourth EF tasks were the day-night and yes-no tasks used in the Wolfe and Bell (2004) study. Lastly, the Dimensional Change Card Sort (DCCS), a measure of rule use and attention-shifting in preschool children, was also administered. Percentage correct scores were calculated for each of the five measures, and a composite WMIC score was computed. Replicating their earlier work, children with higher WMIC scores also had higher PPVT-III scores. Note, however, that their EF tasks included one "hot," or affect-based task (i.e., the goldfish task) and four "cold," or cognitive-based, affect-neutral tasks. In addition, they sampled three different types of inhibitory control. The goldfish activity taxed "hot" EF abilities by involving taste and smell, while the Simon-says-like activity required inhibiting motor responses to verbal instructions, and the day-night and yes-tasks required inhibiting an automatic verbal response. Thus, by reporting data on the WMIC composite, it cannot be determined whether all EF measures were equally related to language performance. It could be that only the cognitive-based measures, as opposed to the affect-based ones, related to language, or that certain types of inhibitory control related better than others. In addition, their method of analysis does not make it possible to know whether the DCCS task alone had any relation to language performance. The current study will attempt to disentangle these measures by examining each $\mathrm{EF}$ area separately.

While Wolfe and Bell $(2004 ; 2007)$ focused on receptive vocabulary as their measure of language ability, other researchers have used expressive vocabulary measures. Gathercole, Service, Hitch, Adams, and Martin (1999) administered the "Oral 
Vocabulary" subtest of the "Word Knowledge" test in the McCarthy Scales of Children's Abilities (McCarthy, 1970) to children between 4;0 and 4;3. Children were verbally given words, which they were asked to define. Definitions were scored as either showing "detailed knowledge," "some knowledge," or "no knowledge" about the word. The authors found that expressive vocabulary scores were related to several working memory measures, including non-word repetition and digit span. Thus, there is some evidence that the relation to working memory holds for both receptive and expressive vocabulary measures.

In summary, correlational studies of EF skills and language abilities in preschoolaged children provide evidence that working memory is linked to some measures of receptive and expressive vocabulary, as well as some comprehensive measures of receptive language. To date, little is known about the direction of the relationship between EF and language. Some possibilities include: (1) better working memory skills facilitate language learning, (2) skilled language learners use strategies, such as selftalk, to regulate attention better than their peers with weaker language abilities, or (3) a third variable, such as socioeconomic status, may affect performance on both measures in similar ways (Wolfe \& Bell, 2007).

\section{Executive Function in Bilinguals and Spanish Speakers}

Executive function in bilingual children. The second population of interest in the current study is bilingual children. Over the last several decades, research examining the effects of bilingualism on cognitive development has revealed that bilinguals show certain advantages compared to monolingual peers. Advantages have been documented in several areas of executive function, particularly in cognitive flexibility. Bialystok (1997) found that bilinguals demonstrated better selective attention 
in the Moving Word Task, where children must remember which printed word form corresponds with each of two pictures, in the face of competing distracters.

In a follow-up study, Bialystok (1999) examined whether the bilingual advantage she previously found in selective attention using a verbal task (Bialystok, 1997) could also be found in the DCCS task, which does not require a verbal response. She studied two groups of preschoolers: a young group (mean age 4) and an older group (mean age 5) with a total of 30 children in each group. Half of the children in each group were bilingual English-Chinese speakers. Chinese was the home language, and English was the community, or school language, but only those bilinguals with near-equal English proficiency compared to monolingual English peers were included in the study. Bialystok found that both young and old bilinguals performed better on the DCCS task compared to their peers. In her discussion of these results, Bialystok argues that "bilingual children are privileged compared to their monolingual peers in their ability to solve problems that are based on conflict and attention" (p.642). These results are relevant to the current study given that some of the L1 Spanish speakers in this sample have some proficiency in English (L2), and thus may score higher on some EF tasks compared to their monolingual peers.

One question that logically follows from the EF findings in bilingual children is: why might bilinguals score better than monolingual peers? One explanation for executive function advantages is that bilinguals have extensive (often daily) practice with inhibitory control in the linguistic domain (Carlson \& Meltzoff, 2008). There is a growing body of evidence showing that bilinguals do not turn each of their languages "on" and "off" during language processing (Guttentag, Haith, Goodman, \& Hauch, 1984). Instead, they must inhibit the non-relevant language during everyday discourse with multiple 
language partners. For example, one scenario is the case of a bilingual child who speaks a language at school that is different from the home language. In recounting the events from school to his parent, the child must inhibit using the school language and instead use the home language. In addition to the linguistic area, Carlson and Meltzoff (2008) point out the bicultural aspect of bilingualism. They suggest that bilinguals practice inhibiting not only their different languages in discourse, but also bilinguals may also have extensive experience inhibiting different social and cultural practices as they interact with individuals from different cultures.

An important caveat when interpreting Bialystok's findings showing bilingual advantages is that no information is reported about the socioeconomic (SES) status of their participants. In their 2007 study, Morton and Harper found no differences between monolinguals and bilingual English-French speakers in Canada on an inhibitory control task, when both groups were matched on ethnicity and socioeconomic status as well as vocabulary, nonverbal intelligence, and "balanced" bilingualism. They criticize the Bialystok $(1997 ; 1999)$ findings because she failed to measure SES. It is possible that the Canadian Chinese-English bilinguals in Bialystok's studies were a higher SES group compared to the monolinguals. Therefore it is unknown whether SES differences that could have accounted for the purported "bilingual" advantage. Given these criticisms surrounding the possible role of SES, the current study collects SES data on all participants and incorporates SES differences into data analyses.

While the work of Bialystok and colleagues is seminal in the field of bilingualism, Carlson and Meltzoff (2008) are the first researchers to study Spanish-English bilinguals in relation to executive function performance. They investigate whether Spanish-English bilinguals show advantages in executive function similar to those reported for bilingual 
speakers with other language pairings (e.g., English-Chinese). Another significant contribution of their work was inclusion of a third population of children, early secondlanguage learners, for purposes of comparing their performance to monolinguals and to "balanced" bilinguals. Carlson and Meltzoff (2008) tested children on conflict tasks (DCCS), delay tasks, and also collected the CBQ inhibitory control scale. They administered a total of nine executive function tasks and calculated a composite EF score, similar to Wolfe and Bell $(2004 ; 2007)$. They included the Expressive One-Word Picture Vocabulary Test to control for verbal ability. They found that bilinguals outperformed both monolinguals and early second-language learners in executive function tasks of working memory and attention-shifting. The second-language learners did not perform differently from the monolingual group. This study extends previous findings from Bialystok's work on balanced bilinguals to a population of Spanish-English bilingual children. More importantly, however, it is among the first to analyze EF performance in early second language learners and show that the bilingual advantage is not found in early stages of second-language acquisition.

Executive function in Spanish speakers. Studies examining executive function in Spanish speakers are extremely limited. The few studies that do exist offer limited information about EF in Spanish speakers. For example, research originating in Spanish-speaking countries may focus on a specific EF area but fails to discuss the findings in terms of Spanish speakers in particular (Leon-Carrion, Garcia-Orza, \& PérezSantamaria, 2004). Other studies report on specific EF tasks, usually with the single goal of providing cross-linguistic validation for use of a task (Matute, Rosselli, Ardila, \& Morales, 2004). Recently, Matute and colleagues (2004) published a neuropsychological test battery, the Evaluación Neuropsicológica Infantil, for Spanish- 
speaking children ages 5-16. This is the first formal test battery to provide culturallysensitive and linguistically-appropriate adaptations of well-known EF tasks used in English speakers for use with children in Mexico. However, to date, there are no published studies reporting use of this test battery with Spanish speakers in the United States.

There exists only one EF study involving Spanish-speaking preschoolers in the United States. McClelland et al. (2007) investigated EF in the context of academic achievement in several populations of preschoolers in Michigan and Oregon. A subset of the Oregon sample of children was Spanish-speaking; therefore all language and EF measures were adapted for use with the Spanish speakers in their sample. The main purpose of their study was to explore relations between behavioral regulation and emergent literacy, vocabulary, and math skills. The authors collected teacher-rated behavior scales, while children were tested in the "Head-to-Toes" task (a measure of inhibitory control, attention, and working memory) and either the English Woodcock Johnson Psycho-Educational Battery-III Tests of Achievement (Woodcock \& Mather, 2000) or the Spanish Bateria Woodcock-Muñoz-R (Woodcock \& Muñoz-Sandoval, 1996). Even though the Spanish-speaking sample in Oregon was from a lower socioeconomic group and had lower vocabulary scores as a group compared to both English-speaking samples, the overall pattern of results did not differ for the Spanish speakers. Children with better behavioral regulation had significantly higher vocabulary scores, math skills, and emergent literacy scores at the start and end of the preschool year. In addition, those children with the greatest gains in behavioral regulation over the school year also had greater gains in emergent literacy, vocabulary, and math compared to their peers with less behavioral regulation growth. These findings provide evidence 
that EF abilities directly relate to academic achievement. Moreover, the patterns of growth in EF, literacy, vocabulary, and math suggest that strengthening EF abilities in particular may result in academic gains across multiple achievement areas.

\section{Language Assessment in Spanish Speakers}

This study will use multiple language measures to obtain a more detailed description about which aspects of language relate to EF abilities. It is well known that language consists of a wide array of skills, including knowledge of the sound system, word structure, vocabulary, and grammar. One of the greatest challenges in assessing language skills of culturally- and linguistically-diverse populations, such as the one being investigated in this study, is the lack of standardized test materials in languages other than English (Langdon, 2008). Researchers in communication disorders caution clinicians about use of standardized language tests that have been adapted from English versions (Bedore \& Peña, 2008). For example, culturally-biased questions (i.e. testing material that is unfamiliar to people of a certain cultural background) can create a skewed picture of an individual's language knowledge. Even well-established, standardized language measures like the Spanish Preschool Language Scale, $4^{\text {th }}$ edition (SPLS-4; Zimmerman, Steiner, \& Pond, 2002b) have been criticized. Gutiérrez-Clellen, Restrepo, and Simón-Cereijido (2006) cited problems with validity of the Spanish grammar test items in the SPLS-4. They argued that rather than testing the structures that are difficult for Spanish-learning children, the SPLS-4 tests grammatical structures that are difficult for English-learning children. While this means that the SPLS-4 may not be tapping into the relevant components of Spanish grammar, it is still the best comprehensive standardized Spanish language measure currently available to clinicians in the United States. 
To further complicate assessment of culturally-and linguistically-diverse populations, there is the additional issue of identifying language delays in children who are bilingual (Bedore \& Peña, 2008). To date, there are no standardized language tests that have been normed on bilingual speakers. Clinically, this means that speechlanguage pathologists (SLPs) often rely on use of different standardized tests for each of the child's two languages. However, norms on these tests are inappropriate given that their use is intended for monolingual speakers. The task of identifying language delay in bilingual speakers is therefore currently not guided by specific clinical cutoffs, but rather relies more heavily on informal observations and clinical judgment. Given lack of a better assessment strategy, in the current study, different standardized tests will be used to assess Spanish versus English knowledge. However, scores are not meant to be interpreted in a standardized fashion. Instead, scores are used solely to measure relative differences in language knowledge among children in this sample.

In order to explore relations between language and EF more broadly, comprehensive language measures were included in the current study. First, the Bilingual Spanish-English version of the Receptive One-Word Picture Vocabulary Test (B-ROWPVT; Brownell, 2000b) was used to replicate previous studies linking receptive vocabulary to EF (Wolfe \& Bell, 2004; Wolfe \& Bell, 2007). Neither the Peabody Picture Vocabulary Test, $3^{\text {rd }}$ ed. (PPVT-III; Dunn \& Dunn, 1997) nor the Spanish-language Test de Vocabulario en Imágenes Peabody (TVIP; Dunn, Lugo, Padilla, \& Dunn, 1986) would adequately measure all participants' receptive vocabularies given that some children were expected to have English language knowledge. Therefore, the B-ROWPVT was administered to obtain an accurate receptive vocabulary measure for all participants. 
The comprehensive language measures selected for the current study include the Expressive Communication Scale of the Spanish Preschool Language Scale, $4^{\text {th }}$ Edition (SPLS-4; Zimmerman et al., 2002b), a standardized comprehensive Spanish language test, and the Expressive Language Scale of the Clinical Evaluation of Language Fundamentals - Preschool Edition (CELF-P; Semel, Wiig, \& Secord, 2004a), a standardized comprehensive assessment of English language. Note that both comprehensive measures in the current study focused exclusively on expressive knowledge. There were two main reasons for this. First, given that language comprehension precedes production skills in typical language development (Fenson et al., 2007), expressive language measures were more likely to provide variability in performance in this sample. Second, preschoolers have shorter attention spans and fatigue more quickly compared to older school-aged children. By administering the expressive component of each comprehensive language measure, over an hour of total language testing was eliminated, allowing for more data collection in EF areas. Finally, one supplemental measure of Spanish grammar, the Spanish Morphosyntax subtest of the Bilingual English Spanish Assessment (BESA SMST; Peña, Gutiérrez-Clellen, Iglesias, Goldstein, \& Bedore, 2007), was included in the test battery.

\section{Focus of the Current Study}

Taken together, research on typically-developing children and children with language or other disorders suggests that executive functioning abilities are related to language in ways that merit further study. In addition, the literature on EF performance in Spanish speakers shows a significant need for research. In examining relations between language and executive function, the current study extends previous work in four ways: 
1. Previous studies examining links between language and EF have used either a single EF task or EF composite scores to correlate to language. This study examined the three EF areas of working memory, inhibitory control, and attention-shifting. In addition, language relations to EF were analyzed separately for the three individual EF areas in order to understand whether all or only some EF areas are related to language.

2. One bias in the executive function literature is the focus on middle-class Englishspeaking populations. The current research examined Spanish speakers who have historically been understudied in the EF literature. Not unexpectedly, the Spanish speakers in this study also come from a lower socioeconomic group compared to that typically reported in EF studies. Thus, this study also extended EF research to a lower SES population.

3. Spanish-speaking preschoolers living in the United States, some of whom inevitably have become exposed to English, are the target population this study. Given this fact, there are potential contributions to the literature showing EF advantages in bilingual children. The literature on bilingualism and EF, however, has also focused on middle-class bilinguals with near-equal proficiency in both of their languages. Several findings are possible in this study. On the one hand, perhaps no EF bilingual advantage will be found since this is a low SES sample. However, it is also possible that the bilingual advantage extends to other SES groups, and thus, that low SES bilinguals may be at an advantage compared to their low SES monolingual peers. If a bilingual advantage is found, this study can further explore which individual EF areas - working memory, inhibitory control, or attention-shifting - show an advantage. 
4. Finally, the main purpose of this study is to examine relations of language to executive function in ways that are more informative to language researchers and clinicians alike. This study is the first to extend language measures beyond the isolated construct of receptive vocabulary. Comprehensive expressive language measures in Spanish and English were analyzed in addition to conceptual receptive vocabulary and Spanish grammar. Together, the three executive function measures and the four language assessments will allow for a more nuanced analysis of language relations to EF. Further, analyses controlled for socioeconomic status in order to account for this potential confound.

\section{Method}

\section{Participants}

Thirty-nine children (18 females, 21 males) participated at age $4 ; 8$ (range $=4 ; 8$ $4 ; 11$ ) as part of an ongoing longitudinal study of Spanish language development at Stanford University directed by Dr. Anne Fernald, Associate Professor of Psychology. Testing took place from July 2008 - June 2009 at a community research facility in East Palo Alto, CA, where participants had completed all prior testing sessions. East Palo Alto is a predominantly low-income community, comprised largely of immigrant families from Mexico. The neighborhood-based research lab is located just a few miles from the Stanford University campus and is staffed by bilingual, bicultural research assistants trained in conducting research with the local Spanish-speaking population.

Participants were initially recruited in $2004-2006$, at age $1 ; 6$, through county birth records granted to Dr. Fernald as part of a 4-year longitudinal study ${ }^{2}$. In this initial and all subsequent testing sessions, parents completed a Basic Information Form (BIF) that asked about the child's health and developmental history, caregiver information, and 
information about parent education and occupation. Exclusionary criteria at age 1;6 included significant complications during pregnancy or birth, serious medical illnesses, hearing loss, and known developmental delays, such as Down syndrome or mental retardation. Spanish language-learning status was determined using the Language Background Questionnaire (LBQ; see procedure outlined below). Children with more than $15 \%$ exposure to a language other than Spanish at age 1;6 were not enrolled in the longitudinal study.

All participants who were initially enrolled at age $1 ; 6$ were called to participate in the current study at $4 ; 8$, regardless of the child's language or developmental status at any subsequent time point. The timing of the sessions was planned with school status in mind, since a marked increase in exposure to English is typical upon entry into kindergarten. None of the children were yet reported to attend kindergarten. Nevertheless, all but four participants were reported to have attended either an early intervention Head Start program or preschool.

Upon completion of both testing sessions for this study, three participants did not meet criteria for inclusion in analyses. One female was excluded due to significant medical history and one male was excluded due to a recently-diagnosed hearing loss. In addition, one male participant with diagnosed language delays came in twice for testing but failed to provide any useable data at either session.

All parents of this final sample of 36 children (17 female, 19 male) re-confirmed at this age point that their children had been full-term with no major complications during pregnancy or birth, and had no known hearing loss or serious illnesses. Parents of two participants reported that their children had been diagnosed with language delay (LD) between age 2;0 and the time of current testing. One LD child was currently receiving 
speech-language therapy twice per week. The second LD child had just recently been diagnosed but had not yet begun receiving speech-language services. However, none of the LD children had general cognitive delays or other serious medical conditions. Their LD status alone was not considered exclusionary in this study. On the contrary, for purposes of examining the relation between language and executive function, these children would likely provide valuable information about the low-scoring end of the language ability spectrum.

Almost half of the children (17 of 36) were first-born, a quarter (9 of 36) were second-born, and the remainder (10 of 36$)$ were third- to fifth-born children.

Socioeconomic status of the participants was derived using the Hollingshead Four Factor Index of Socioeconomic Status (HI; Hollingshead, 1975; see below). Participant age at test, sex, birth order, and socioeconomic status (HI) are provided in Table 1.

Table 1 Demographic information for all participants

\begin{tabular}{ccccccl}
\hline Participant & Age & Sex & $\begin{array}{c}\text { Birth } \\
\text { Order }\end{array}$ & HI $^{\mathrm{a}}$ & $\begin{array}{c}\text { Spanish } \\
\text { Exposure }^{\mathrm{b}}\end{array}$ & Language Status $^{\mathrm{c}}$ \\
\hline 1 & $4 ; 8$ & $\mathrm{~F}$ & 1 & 16 & 100 & Primarily Spanish \\
2 & $4 ; 8$ & $\mathrm{~F}$ & 1 & 19 & 96.6 & Primarily Spanish \\
3 & $4 ; 8$ & $\mathrm{M}$ & 1 & 38 & 91.9 & Primarily Spanish \\
4 & $4 ; 8$ & $\mathrm{~F}$ & 3 & 26 & 91 & Primarily Spanish \\
5 & $4 ; 8$ & $\mathrm{~F}$ & 1 & 58 & 90.1 & Primarily Spanish \\
6 & $4 ; 8$ & $\mathrm{~F}$ & 1 & 32 & 90 & Primarily Spanish \\
7 & $4 ; 9$ & $\mathrm{~F}$ & 1 & 63 & 89.6 & Primarily Spanish \\
8 & $4 ; 8$ & $\mathrm{M}$ & 1 & 13 & 83.3 & Primarily Spanish \\
9 & $4 ; 9$ & $\mathrm{M}$ & 1 & 45 & 82.3 & Primarily Spanish \\
10 & $4 ; 8$ & $\mathrm{~F}$ & 2 & 15 & 81.8 & Primarily Spanish \\
11 & $4 ; 8$ & $\mathrm{~F}$ & 2 & 15 & 81.6 & Primarily Spanish \\
\hline
\end{tabular}




\begin{tabular}{|c|c|c|c|c|c|c|}
\hline 12 & $4 ; 8$ & $M$ & 2 & 13 & 77.5 & Primarily Spanish \\
\hline 13 & $4 ; 8$ & $M$ & 1 & 25 & 76.8 & Primarily Spanish \\
\hline 14 & $4 ; 8$ & $M$ & 1 & 29 & 75.7 & Primarily Spanish \\
\hline 15 & $4 ; 10$ & $M$ & 1 & 38 & 74.7 & Primarily Spanish \\
\hline 16 & $4 ; 8$ & $\mathrm{~F}$ & 2 & 16 & 74.5 & Primarily Spanish \\
\hline 17 & $4 ; 10$ & $\mathrm{~F}$ & 3 & 16 & 73.3 & Primarily Spanish \\
\hline 18 & $4 ; 11$ & M & 1 & 16 & 72 & Primarily Spanish \\
\hline 19 & $4 ; 8$ & $M$ & 1 & 53 & 70.6 & Spanish-English ${ }^{\star}$ \\
\hline 20 & $4 ; 8$ & $F$ & 1 & 27 & 66.1 & Spanish-English \\
\hline 21 & $4 ; 10$ & $M$ & 2 & 8 & 65.4 & Spanish-English \\
\hline 22 & $4 ; 8$ & $M$ & 2 & 25 & 64.3 & Spanish-English \\
\hline 23 & $4 ; 8$ & $F$ & 1 & 25 & 63.2 & Spanish-English \\
\hline 24 & $4 ; 8$ & $\mathrm{~F}$ & 1 & 25 & 63.2 & Spanish-English \\
\hline 25 & $4 ; 8$ & $\mathrm{~F}$ & 4 & 13 & 62.7 & Spanish-English \\
\hline 26 & $4 ; 8$ & $M$ & 2 & 13 & 58.9 & Spanish-English \\
\hline 27 & $4 ; 8$ & $M$ & 3 & 23 & 57.7 & Spanish-English \\
\hline 28 & $4 ; 8$ & $\mathrm{~F}$ & 3 & 21 & 55.6 & Spanish-English \\
\hline 29 & $4 ; 11$ & $M$ & 2 & 34 & 50.1 & Spanish-English \\
\hline 30 & $4 ; 9$ & $M$ & 1 & 30 & 49.4 & Spanish-English \\
\hline 31 & $4 ; 8$ & $M$ & 2 & 22 & 49.2 & Spanish-English \\
\hline 32 & $4 ; 9$ & $\mathrm{~F}$ & 3 & 63 & 44.5 & Spanish-English \\
\hline 33 & $4 ; 8$ & $\mathrm{~F}$ & 5 & 16 & 34.3 & Spanish-English \\
\hline 34 & $4 ; 9$ & $M$ & 3 & 13 & 32.8 & Spanish-English \\
\hline 35 & $4 ; 8$ & $M$ & 3 & 8 & 32.3 & Spanish-English \\
\hline 36 & $4 ; 9$ & $M$ & 3 & 13 & -- & Spanish-English \\
\hline Notes: & \multicolumn{6}{|c|}{$\begin{array}{l}\text { a Score on the Hollingshead Four Factor Index of Social Status (range=8-66) } \\
\text { bercentage of total language exposure to Spanish, based on the Language } \\
\text { Background Questionnaire (LBQ). Datum is missing for one family that failed to } \\
\text { complete the LBQ. } \\
\text { "Using a median split, "Primarily Spanish" language status was assigned to those } \\
\text { participants with relatively more exposure to Spanish and less exposure to }\end{array}$} \\
\hline
\end{tabular}


English. Spanish-English learners are those participants with relatively more English exposure. Status for one participant with missing language exposure information was made based on examiner observations of his language knowledge and on a general description of the child's language environment.

- Participant met "Spanish-English Learner" status for the first time since initial enrollment in the longitudinal study at age $1 ; 6$.

\section{Measures}

Language learning status. Following Marchman, Martínez-Sussmann, and Dale (2004), parents' responses on the LBQ were used to quantify exposure to each language. Detailed information was gathered about the child's daily schedule, amount of time spent with different people (e.g., caregivers, preschool teachers and peers, siblings, etc.), and languages used by each speaker when interacting with the child. The total number of hours of English and Spanish heard each week were calculated in order to compute the percentage of exposure to each language. The Spanish-English exposure proportion for each participant is listed in Table 1.

Recall that at induction at age $1 ; 6$, all children could be considered primarily Spanish learners. The criteria for a "Primarily Spanish Learner" were that (1) Spanish was the primary language spoken in the home, and (2) children were not exposed to more than $15 \%$ English in their daily activities. However, over the course of children's participation in the longitudinal study from one to four years of age, these preschoolaged children were exposed to considerably more English as a group than at induction, and further, the degree of exposure to English may have varied across time for some children. Therefore, at the time of current testing, most children no longer met the initial criteria for being considered primarily Spanish-language learners.

There was nevertheless still a considerable range in the degree of Spanish and English exposure in this group of children. Thus, for the purposes of some analyses, the sample of 36 children was divided into two groups based on a median split of language 
exposure levels. The top half of the distribution included those children with relatively more exposure to Spanish compared to their peers in this sample $(n=18)-$ those children in the group most similar to their language status at induction have remained "Primarily Spanish Learners." The bottom half of the distribution $(n=18)$ were those children who, compared to the rest of the group, experienced relatively more English exposure and relatively less exposure to Spanish. These children were grouped as "Spanish-English Learners."

For the Primarily-Spanish Learners, the range of percentage exposure to Spanish was $72-100(M=83.5, S D=8.5)$, while for the Spanish-English Learners, the range was $32.3-70.6 \%(M=54.1 ; S D=12.3)$. Note that all children as a group are still best described as learning more Spanish than English. While the groupings defined here arbitrarily placed children into groups based on the characteristic of this particular sample, they allowed the analyses to explore variation in degree of exposure to English on language and executive function outcomes.

Language exposure information used in the current study was based on the LBQ for 35 of 36 participants, as shown in Table 1. LBQ info was unavailable for one remaining participant, but he was classified as a Spanish-English Learner based on examiner observation and on information informally discussed with the parent. Given that longitudinal language exposure data available for all children, it was found that for most children in the Spanish-English Learner group (12 of 18), an increase in the degree of exposure to English typically occurred in the year just prior to the current testing point. This is also indicated in Table 1.

Socioeconomic status. In order to better characterize the demographics of this sample and to understand how results from this study may generalize to other 
populations, parent education levels from the BIF were used to calculate the Hollingshead Four Factor Index of Social Status (HI; Hollingshead, 1975) for each family. Moreover, several previous studies have shown that socioeconomic status correlates with performance on both language assessments (Arriaga, Fenson, Cronan, \& Pethick, 1998; Dollaghan et al., 1999) and executive function tasks (Ardila, Rosselli, Matute, \& Guajardo, 2005; Carlson \& Meltzoff, 2008; Hughes \& Ensor, 2005; Morton \& Harper, 2007). The $\mathrm{HI}$ ranges from a score of 8 to 66 , grouping scores into SES categories that identify five social strata: unskilled laborers $(\mathrm{HI}=8-19)$, semi-skilled workers $(H \mathrm{I}=20-29)$, skilled/clerical workers $(\mathrm{HI}=30-39)$, minor professionals $(\mathrm{HI}=40-54)$, and major professionals $(H \mathrm{H}=55+)$.

The mean $\mathrm{HI}$ for all participants in the current study was 25.7 (SD=14.9). The majority of families (over $70 \%$ ) fell in the lowest two social strata, and only five families (less than 15\%) scored in the top two strata. Thus, the sample is best described as predominantly low SES. Note that the Hollingshead Index did not differ between the two language subgroups (Primarily Spanish Learners: $M=27.3, S D=15.5$; Spanish-English Learners: $M=24.0, S D=14.5 ; p>0.40$ ).

Nonverbal cognition. The brief nonverbal IQ screener from the Leiter International Performance Scale-Revised (Leiter-R; Roid \& Miller, 1997) was used to measure cognitive ability. The Leiter-R is a well-known, standardized measure of fluid intelligence designed for use with children ages $2 ; 0-20 ; 11$. Unlike other intelligence measures, the Leiter-R does not require use of spoken or written language during administration. Leiter-R scores were obtained for 32 of 36 participants, with no child scoring below a standard score of 83 . One score was missing due to examiner error, and three were missing because the testing session was terminated due to child fatigue. 
However, none of the children with missing Leiter-R scores were excluded from analyses given that they performed well within the normal range on standardized language assessments and that there was no evidence of suspected cognitive delays (e.g., parent concerns or examiner observations). The mean Leiter-R standard score for all participants was $100.9(S D=12.3)$. Mean scores for the Primarily Spanish Learners $(n=18, M=99.8, S D=13.8)$ and the Spanish-English Learners $(n=14, M=102.2, S D=10.3)$ were not significantly different from each other $(p>0.40)$. Thus, this sample includes only children with typical-range non-verbal intelligence.

Executive function. Executive function (EF) was assessed using three face-toface tasks and one parent-report measure. Tasks that tapped into working memory, inhibitory control, and attention-shifting were administered to each participant. The working memory measure is standardized for English-speaking children only, and the inhibitory control and attention shifting measures are not standardized on any population. This lack of norms, however, is not surprising given the known difficulties with standardizing most EF measures (Banich, 2009). Nevertheless, all activities chosen to sample the various EF components are appropriate for preschool-aged children and their use is well-documented in the research literature (Carlson, 2005).

Digit span. To obtain a measure of working memory, the "Number Memory Forward" subtest of the Test of Auditory Processing Skills, Third Edition (TAPS-3; Martin \& Brownell, 2005) was administered. The TAPS-3 is a standardized, norm-referenced test for use with English-speaking children ages 4;0-18;11. The main purpose of the TAPS-3 is to provide a comprehensive assessment of children's ability to perceive and manipulate auditory stimuli. The test consists of several different subtests sampling the areas of auditory discrimination, memory, and comprehension and reasoning. For the 
current study, however, only the "Number Memory Forward" subtest was selected given that this is the test in the TAPS-3 battery that measures verbal working memory.

The subtest was administered as is described in the TAPS-3 examiner's manual., with the exception that children were given a choice of completing the test in Spanish or English. While there does exist a Test of Auditory Processing Skills, Third Edition Spanish Bilingual Version (Martin, 2005), norms on this test are not available until age 5;0. Therefore, the TAPS-3 Number Memory Forward subtest was used for all children, regardless of their language of choice. To select the language for administration, children were asked whether they wanted to play the "number game" in Spanish or English. This option was given because past research experiences with this population have revealed that even monolingual or Spanish-dominant bilingual children often experience numbers in English-only contexts. This may be because parents try to teach counting in English (even if the parents have English knowledge limited to counting, the alphabet, or color words) or because children are exposed to English number words through toys, television, or older children. Sixteen children chose to complete this task in English, and twenty children preferred to complete it in Spanish.

Children were instructed to repeat increasingly longer lists of numbers (digits 1-9) in exactly the order they were presented. The test begins with two items testing 2-digit sequences, then two items testing 3-digit sequences, two items testing 4-digit sequences, and so on. A maximum raw score of 2 was possible for each item if all digits were repeated in the correct order. Children could receive one point on an item if they repeated all the digits but not in the correct order. A raw score of 0 meant that children were unable to repeat 2-digit sequences reliably. A raw score of 4 corresponds to a ceiling at 2-digit sequences, a raw score of 8 to ceiling at 3-digit sequences, a raw score 
of 12 to 4-digit sequences, etc. Standard scores could not be obtained, given that some children completed the test in English and others in Spanish, but that norms are only available for the English administration. For this reason, raw scores are reported in all data analyses.

Day-night task. An abbreviated version of the original "day-night" task (Gerstadt, Hong, \& Diamond, 1994) was used as a measure of inhibitory control - the ability to suppress an automatic response. Gerstadt et al. designed this task as a child version of the standardized adult Stroop Color and Word Test (Golden, 2002) that does not rely on reading. Children were first asked about when the sun comes up (during the day) and when the moon comes up (during the night). The examiner showed the child a white card with a picture of a sun and a black card with a picture of the moon (see Figure 1). The child was instructed to say "night" when shown the sun card, and "day" when shown the moon card. In other words, the urge to label each card according to its contents had to be suppressed, and instead the opposite answer had to be given. Given that this was a measure of inhibitory control, self-corrected responses (e.g., child responded "day...I mean, night!") were scored as incorrect. The order of presentation for sun and moon cards matched the first 8 trials described in Gerstadt et al. Accuracy on the task was recorded as percent correct out of 8 trials.
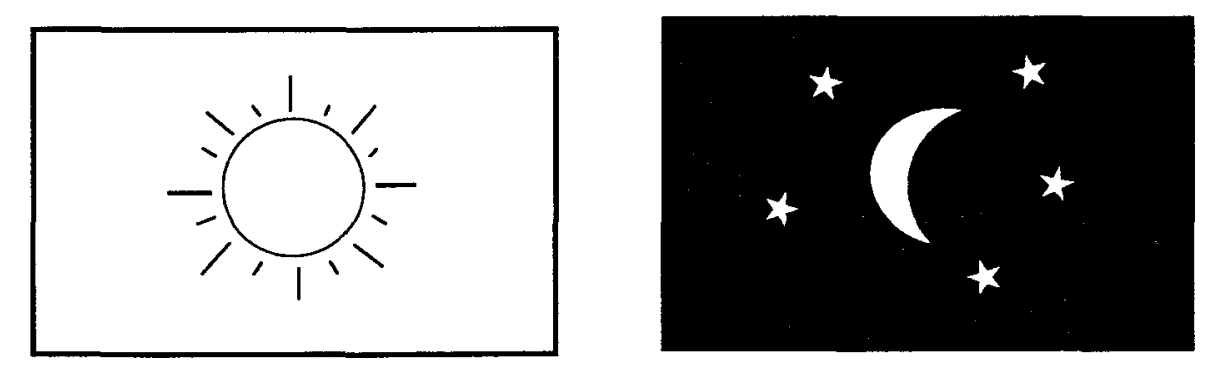

Figure 1 Stimulus cards used in the day-night task 
Standard Dimensional Change Card Sort (Standard DCCS). The Standard DCCS task is a classic measure of ability to shift attention, or "mental set," in preschoolaged children (Zelazo et al., 1996). The research literature on the Standard DCCS shows that monolingual English-speaking three-year-olds reliably fail the task (Carlson, 2005; Frye, Zelazo, \& Palfai, 1995; Kirkham, Cruess, \& Diamond, 2003; Zelazo et al., 1996). However, the probability of passing the task increases significantly once children enter the 4-year-old range. Some studies have found that young 4-year-olds pass but still struggle with the DCCS task, with passing rates around 60\% (Frye et al., 1995). Kirkham et al., however, reported a passing rate of $92 \%$ in a sample of predominantly young 4-year-olds ( $M=4 ; 1)$ of European Caucasian descent from middle class homes. Passing rates for older 4-year-olds are consistently high, ranging from $76 \%$ (Carlson, 2005) to $90 \%$ (Zelazo et al., 1996). Together, these studies suggest that children begin passing the task at age 4 and master it before reaching age 5 . Thus, this task is developmentally appropriate for use in the current study.

Children were told they were going to play a game with some cards. The children saw rectangular boxes in front of them, one with a card showing a red cat and the other with a card showing a blue horse. The examiner then handed the child a set of cards picturing red horses and blue cats (see Figure 2). 
Sorting boxes with a red cat model card and a blue horse model card
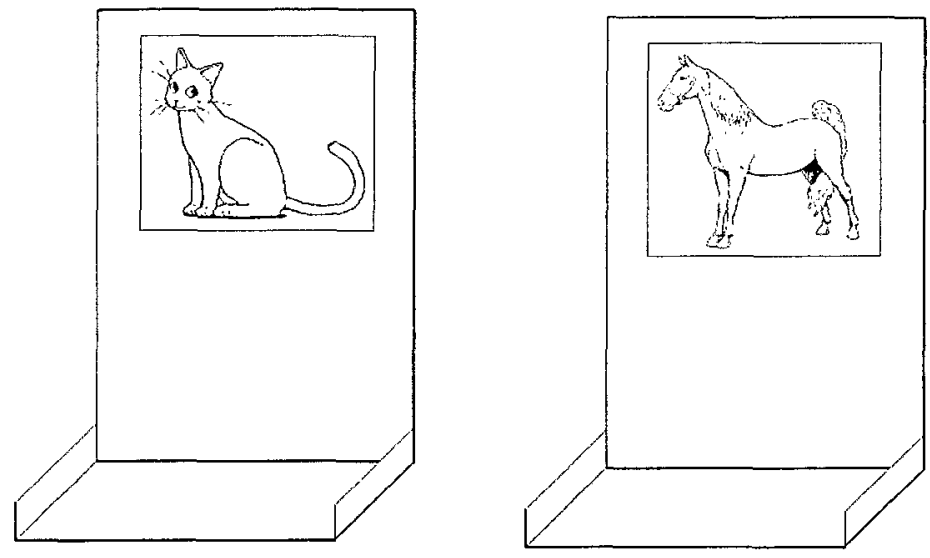

Red horse and blue cat sorting cards
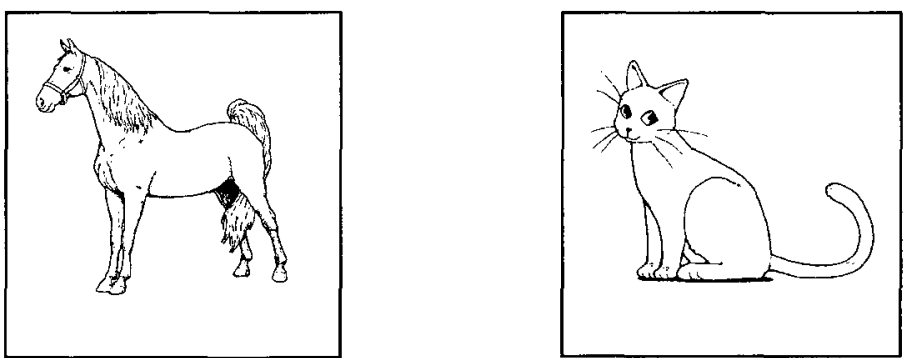

Figure 2 Sorting boxes, model cards, and sorting cards used in the Standard $D C C S$ task. For the purposes of this illustration, red cards are depicted in dark grey and blue cards are depicted in white.

Children were first instructed to "play the color game" by putting all of the red cards in the box with the red cat and all of the blue cards in the box with the blue horse. Children had to sort six cards correctly on this task in order to pass the initial sorting test. If a child did not sort six cards correctly, additional cards were shown until the child passed the initial sorting test. Once the child passed, the examiner changed the rules of the game, announcing that they were now going to "play the animal game" and put all cats in the box with the red cat and all horses in the box with the blue horse. Children were then tested on another set of six cards. 
The task was administered exactly as presented in Kirkham, Cruess, and Diamond (2003), except for minor changes to the picture stimuli. In the Kirkham et al. study, children saw trucks and stars (instead of cats and horses) and were instructed to play color and shape games (instead of color and animal games). In modifying this task for use with Latino preschoolers, the stimuli were changed to ensure the task was culturally appropriate for this participant sample. While playing the "shape game" is not uncommon in typical middle-class American homes, it is not necessarily a familiar routine for most Latino preschool children. For this reason, the "animal" dimension was selected in place of the "shape" dimension. Pilot testing in June 2008 revealed that children sorted by the color and animal dimensions equally well.

In order to succeed at the Standard DCCS task, children had to shift mental sets and attend to the animal dimension instead of the color dimension. Children were scored according to the pass-fail criteria outlined in Kirkham et al. (2003). Children passed the task if they sorted either 5 or 6 of the final 6 cards correctly. Any score under 5 was considered failure to sort by the new rule.

Children's Behavior Questionnaire (CBQ; Rothbart et al., 2001). In addition to the three face-to-face tasks, a parent-report measure of inhibitory control was obtained through the CBQ. The official Spanish translation of the CBQ was used in this study, with minor changes in wording made throughout to account for vocabulary differences between Castilian Spanish and Mexican Spanish spoken by the participants in the current study. All wording changes were made in consultation with two native Mexican Spanish speakers, including a post-doctoral researcher and director of research studies at the EPA testing center. 
Completion of the questionnaire involved rating 13 statements using a 7-point scale ranging from "extremely untrue" to "extremely true." Some sample CBQ statements included: "Approaches places that $\mathrm{s} /$ he has been told are dangerous slowly and cautiously" and "Is usually able to resist temptation when told s/he is not supposed to do something." Note that CBQ statements sampled a mix of "cold" and "hot" EF scenarios involving inhibitory control. Following the $C B Q$ scoring guidelines, ratings on these statements allowed for calculation of an inhibitory control (IC) index. The maximum IC score possible was 7.00 and the lowest was 1.00 . Higher scores (those closer to 7.00 ) indicated better inhibitory control.

Language skills. Four assessments were administered to measure language abilities. Three norm-referenced, standardized tests were used: one test of vocabulary and two tests of comprehensive language. Lastly, a separate test of Spanish grammar knowledge was administered. Each of the measures is described in more detail below.

\section{Receptive One-Word Picture Vocabulary Test, Spanish-English Bilingual}

Edition (B-ROWPVT; Brownell, 2000b). The B-ROWPVT is a test of receptive vocabulary designed for use with children ages $4 ; 0$ to $12 ; 11$ who have varying levels of proficiency in English and Spanish. The test was administered exactly as is described in the examiner's manual. The child was shown a page with four horizontally-arranged color pictures. The examiner then asked the child "Show me $x$." where $x$ is a word such as "spoon" or "running," and the child pointed to the appropriate picture. Each child was first prompted in his/her dominant language. If the child responded incorrectly, then the question was repeated in his/her non-dominant language. Only if the child responded incorrectly to prompts in both languages was an item scored as incorrect. In this way, the B-ROWPVT allows examiners to obtain a "conceptual vocabulary" score that is 
independent of the language in which a child knows a particular test item. Standard scores were obtained from this test.

\section{Spanish Preschool Language Scale, $4^{\text {th }}$ edition - expressive} communication scale (SPLS-4; Zimmerman et al., 2002b). The SPLS-4 is a wellknown and widely used test of comprehensive language in Spanish-speaking children ages $0 ; 0-6 ; 11$. This test was administered to obtain a comprehensive expressive language index in Spanish for each child, beyond vocabulary (B-ROWPVT) alone. In the SPLS-4, a test booklet with pictures is used to probe several language areas, including understanding and production of pronouns, spatial prepositions, and colors, as well as ability to comprehend and re-tell a story. Standard scores were computed for all children following specified procedures. The "Auditory Comprehension Scale" of the SPLS-4 was not administered due to constraints in the amount of testing 4-year-olds could tolerate. Given that all children had lifelong exposure to Spanish, and that language comprehension precedes production skills in typical language development (Fenson et al., 2007), the "Expressive Communication Scale" was thought more likely to provide variability in performance as compared to a receptive language scale in this sample of children.

\section{Clinical Evaluation of Language Fundamentals - Preschool Edition $2^{\text {nd }}$} edition, expressive language scale (CELF-P; Semel et al., 2004a). Similar to the SPLS-4, this norm-referenced comprehensive language assessment is designed for use with children ages $3 ; 0$ to $6 ; 11$. It samples comprehension and production of grammatical structures, as well as expressive vocabulary and ability to recall sentences in English. Clinically, it is designed for use with monolingual learners of English; therefore, the standard scores obtained from this administration are not intended for interpretation in a 
standardized manner. In other words, presence or absence of a language disorder cannot be ascertained from participants' performance on this test in this sample of children. In this study, children were administered the CELF-P because it was known that some children would be learning English at the time of current testing. For this reason, full-scale language assessments were administered in English as well as Spanish to obtain a comprehensive assessment in both of the languages that these children were learning. However, the standard scores obtained on the English CELF-P should be interpreted with caution.

\section{The Bilingual English-Spanish Assessment, Spanish morphosyntax} subtest (BESA SMST; Peña et al., 2007). The Bilingual English Spanish Assessment (BESA) is a comprehensive battery of language tests examining phonology, morphology, semantics, syntax, and pragmatics in Spanish-English bilingual children starting at 4 years of age (Peña et al., 2007). Its main goal is to identify bilingual children with SLI by testing those language structures that pose the greatest difficulty for Spanish speakers with language delay. The test is not yet commercially available, as norming and standardization is still in progress. However, one of the test developers provided an experimental version of the BESA for use in this study and other research projects.

The BESA SMST, one subtest in the BESA battery, was used to obtain information about children's expressive Spanish morphosyntax skills. It was included to expand the assessment of children's language knowledge beyond the language areas sampled by the SPLS-4. This was included to address concerns with validity of the Spanish grammar test items in the SPLS-4 (Gutiérrez-Clellen, Restrepo, \& SimónCereijido, 2006). In the current study, if no relation is found between performance on the SPLS-4 and measures of executive function, one explanation may be that the SPLS-4 
fails to sample the appropriate Spanish grammar areas. For that reason, the BESA SMST was included as an additional data point to consider in the case that no relation is found using the SPLS-4. Again, its purpose was not to serve as a diagnostic marker of SLI.

The BESA SMST consists of a "Sentence Completion" subsection and a "Sentence Repetition" subsection. All responses in the "Sentence Completion" were obtained through elicited production or cloze tasks while looking at a test booklet of pictures. For example, children were shown a picture of a cat pushing dishes off of a table and heard "El gato está tirando los platos al suelo. Y aqui, ¿qué esta tirando el gato?" (The cat is pushing the plates (off the table) onto the floor. And here, what is the cat pushing?), to which the child had to respond "las manzanas" (the apples, with "the" appropriately marked for gender and number). The maximum raw score on this section was 23.

In the "Sentence Repetition" subsection, children heard increasingly longer and more grammatically complex sentences, and they were instructed to repeat the sentences exactly as they heard them. Children could receive a maximum raw score of 51 on the "Sentence Repetition" subsection. A composite BESA SMST score was used for analyses in the current study. The composite was computed by calculating a simple average of the "Sentence Completion" and "Sentence Repetition" percent correct scores. Reliability

All children were video-recorded during each testing session to ensure accuracy of test scoring. The author of this paper originally scored all test protocols and questionnaires. Then, a highly-experienced research assistant re-checked scoring on all tasks administered. For standardized tests, this included checking that raw scores were 
added correctly and that conversions to standard scores and percentiles were done using the appropriate norms and conversion tables in the scoring manuals. In the case of non-standardized tasks (e.g., BESA SMST, digit span, day night, Standard DCCS, and the $\mathrm{CBQ}$ questionnaire), data entry of raw scores was verified, and percent correct scores were automatically calculated. All scoring discrepancies were reviewed by the author and corrected as necessary.

Seventy-two testing sessions were conducted for the 36 participants in this study. The author conducted 69 of 72 sessions (96\%), and a highly-experienced research assistant conducted the remaining three sessions. Videos of those three testing sessions were viewed by the author to ensure consistency in test administration and scoring of responses.

\section{Procedure}

Families participating in the current study were scheduled for two testing sessions, each lasting up to two hours. Accommodations for play or snack breaks were made as the parent and examiner deemed appropriate during sessions. Upon arrival at the facility, the examiner explained all testing procedures, questionnaires, and consent forms to the parent. While the parent completed the forms, the examiner played with the child to help establish rapport. Next, the child watched a 5-minute video as part of their participation in the larger longitudinal study. Then, the child sat with the examiner in a child-friendly testing room, while the parent either observed in the background or stayed in a waiting area. Parents were encouraged to choose whichever option would be best for the child's performance.

Testing was distributed over two sessions, approximately one week apart. During the first day of testing, the B-ROWPVT, the expressive language scale of the 
SPLS-4, and the BESA SMST were administered, together with the "day-night" executive function task. At the second testing session, the CELF-P and the brief nonverbal IQ screener of the Leiter-R were administered, together with the digit span and Standard DCCS tasks. To maintain confidentiality, all children's test protocols and video-recordings were identified by number, not by name. At the conclusion of each testing session (two sessions total), families received a $\$ 50$ gift card in appreciation of their time. This compensation was made possible by an NIH grant to Dr. Anne Fernald (1RO1DC00838).

\section{Results}

In order to answer the main research question examining relations between language and executive function performance, results are presented in four main sections. First, descriptive statistics on the four executive function measures are presented. Analyses focus on the sample as a whole, as well as on the PrimarilySpanish and Spanish-English learner groups separately. Intercorrelations among the EF measures reveal to what degree each task measured different subcomponents of the executive function umbrella. Second, scores on the language measures in this sample of preschoolers are described, again looking at overall performance of the entire cohort, as well as the Primarily-Spanish and Spanish-English learner groups separately. Intercorrelations among the language measures are presented to explore relations between children's scores on the four different language assessments. In the third section, relations between the Hollingshead Index of Socioeconomic Status and tests of both language and executive function are presented. These analyses are critical to understanding whether any links between language and EF measures are driven by parallel relations to socioeconomic status. 
Finally, analyses in the fourth section focus on the main question: Which measures of language are most strongly correlated with executive function? If the findings replicate previous links between receptive vocabulary and working memory and inhibitory control (Wolfe \& Bell, 2007), correlations between the B-ROWPVT and the digit span, day-night, or CBQ index should be significant, even when controlling for SES. Yet, recall that the current study extends previous work in two ways. First, language skills were assessed more broadly - beyond receptive vocabulary alone - by administering comprehensive expressive language measures (the Spanish-language SPLS-4 and the English-language CELF-P). If comprehensive language scores, like receptive vocabulary, also relate to executive function, then correlations between the SPLS-4 and the EF measures, and between the CELF-P and the EF measures should also be significant. Second, recall that executive function abilities were examined more broadly as well - beyond measures of working memory and inhibitory control - by also administering a measure of attention-shifting (the Standard DCCS). Because the Standard DCCS task yields a binary pass-fail outcome, children are grouped into outcome groups: those who passed versus those who failed the Standard DCCS task. If children with greater attention-shifting abilities also have better language skills, then children who passed the Standard DCCS should have significantly higher scores on measures of vocabulary and/or language compared to children who failed the Stanford DCCS task.

\section{Executive Function Measures}

Overall performance. In this section, performance is described for the working memory and inhibitory control measures, both of which were continuous variables (i.e., any value within the range was possible). The Standard DCCS task, however, yielded a 
binary pass-fail outcome; therefore, summary measures for this task are shown separately. Correlational analyses are used to examine relations among the working memory and inhibitory measures only, given that such analyses are not appropriate for binary variables.

Working memory and inhibitory control. Performance on the digit span, daynight, and CBQ inhibitory control index is summarized in Table 2. Descriptive statistics are reported for all participants together, as well as for the Primarily Spanish Learner and Spanish-English Learner groups separately. There was considerable variability in all measures and for all groups. No significant differences in performance were found between the Primarily Spanish Learner and Spanish-English Learner groups on any task shown in Table 2 (all $p>.20$ ).

Table 2 Descriptive statistics for working memory and inhibitory control measures

\begin{tabular}{|c|c|c|c|c|c|c|}
\hline & \multicolumn{2}{|c|}{$\begin{array}{c}\text { All participants } \\
(n=36)\end{array}$} & \multicolumn{2}{|c|}{$\begin{array}{l}\text { Primarily Spanish } \\
\text { Learners }(n=18)\end{array}$} & \multicolumn{2}{|c|}{$\begin{array}{l}\text { Spanish-English } \\
\text { Learners ( } n=18)\end{array}$} \\
\hline & Mean (SD) & Range & Mean (SD) & Range & Mean (SD) & Range \\
\hline \multicolumn{7}{|l|}{ Working Memory } \\
\hline Digit Span Raw Score ${ }^{a}$ & $9.2(3.3)$ & $0-16$ & $8.9(3.3)$ & $0-16$ & $9.4(3.3)$ & $4-16$ \\
\hline \multicolumn{7}{|l|}{ Inhibitory Control } \\
\hline Day-Night $(\%)^{D}$ & $78.3(19.4)$ & $25-100$ & $74.5(22.5)$ & $25-100$ & $82.2(15.5)$ & $50-100$ \\
\hline$C B Q: I C$ index ${ }^{c}$ & $4.77(0.75)$ & $3.23-6.23$ & $4.58(0.74)$ & $3.23-6.23$ & $4.96(0.72$ & $3.23-6.17$ \\
\hline \multicolumn{7}{|c|}{$\begin{array}{l}\text { Notes. }{ }^{\text {a }} \text { Children with a raw score of } 0 \text { were unable to repeat 2-digit sequences. A raw score of } 4 \\
\text { corresponds to a ceiling at 2-digit sequences, a raw score of } 8 \text { to ceiling at 3-digit sequences, } \\
\text { a raw score of } 12 \text { to } 4 \text {-digit sequences, etc. } \\
\text { o Percent correct, out of } 8 \text { possible test trials. } \\
\text { 'Mean score on the inhibitory control index of the Children's Behavior Questionnaire (CBQ). } \\
\text { Index ranges from } 1.00 \text { (least inhibitory control) to } 7.00 \text { (most inhibitory control). }\end{array}$} \\
\hline
\end{tabular}


reflecting variation in performance on this measure. On average, children reached their ceiling working memory capacity between 3 - and 4 -digit-long sequences of numbers. In other words, children's verbal working memory, as indexed by the digit span task, spanned on average 3 or 4 units of information before experiencing breakdowns in capacity. Recall that children were given a choice of completing the digit span task in either Spanish or English. In order to ensure that there were no group differences between those children who did the task in Spanish ( $n=20$; raw scores: $M=8.5 ; S D=$ 3.6) and those who completed it in English ( $n=16$; raw scores: $M=10.0 ; S D=2.8$ ), an independent samples t-test was conducted. Results revealed that there were no differences between the two groups $(p>.18)$.

While children performed below age-based expectations on the digit span task, mean percentage correct on the day-night task was $78 \%$. In general, children were performing well on this task, inhibiting automatic responses on more than three-fourths of test trials. This level of performance on the abbreviated day-night version is comparable to the findings reported for this age group using the original day-night task from Gerstadt et al. (1994).

Lastly, the CBQ inhibitory control index showed that in general children were rated by their parents as having average inhibitory control abilities. The index scores in the current sample were comparable with those found by Wolfe and Bell (2004) in a sample of English-speaking children ages $4 ; 4-4 ; 8$. It should be noted that a surprising majority of the children in this sample were rated by their parents as having average levels of inhibitory control.

Attention-shifting. Performance on the Standard DCCS task is shown separately, in Figure 3. Recall that the Standard DCCS task was used as a measure of 
attention-shifting and yielded a pass or fail outcome. Figure 3 shows the proportion of children who achieved passing criterion for all participants together (left bar), as well as for the Primarily Spanish Learner (middle bar) and Spanish-English Learner (right bar) groups separately. While it appears that a higher percentage of Spanish-English Learners passed the Standard DCCS, chi-square tests revealed that the distribution of scores was not significantly different for the Primarily Spanish Learners and for the Spanish-English Learners $\left(c^{2}(, N=36)=1.08, p>0.40\right)$.



Figure 3 Percentage of participants who passed the Standard DCCS task. Percentages are reported for all participants $(n=36)$, and for Primarily Spanish Learners $(n=18)$ and Spanish-English Learners $(n=18)$ separately. The pass-fail distributions on the Standard DCCS did not differ by language groups, $c^{2}(1, N=36)=1.08, p>0.40$.

The Standard DCCS literature has reported that 3-year-old English monolingual speakers reliably fail the task, whereas 4-year-olds pass it (Carlson, 2005; Frye et al., 1995; Kirkham et al., 2003; Zelazo et al., 1996). Although one study examined performance of 15 bilingual English-Chinese children on the Standard DCCS (Bialystok, 1999), the children ranged in age from 3;2-4;9. Given the large age range and the 
known failure rate in 3-year-old monolingual children, it is difficult to interpret Bialystok's (1999) mean passing rate of $69 \%(S D=36 \%)$. However, compared to previous studies examining performance of 4-year-old monolingual English-learning children, a smaller proportion of children achieved passing criterion in this study. In this population, the passing rate was approximately $10 \%$ lower than the lowest rate reported in the EF metaanalysis by Carlson (2005). The current study, however, differs from classic studies examining performance in Standard DCCS in several ways that may account for differences between the current study and the existing literature on the Standard DCCS task. These differences are presented further in the discussion.

Intercorrelations among executive function measures. Intercorrelations between digit span scores (working memory) and percent-correct scores on the daynight task (inhibitory control) were examined. The executive function measures in this study were not significantly intercorrelated. Table 3 shows Pearson correlation coefficients for working memory, response inhibition, and the parent-report measure of inhibitory control for all participants $(n=36)$. Subsequent analyses revealed no differences between the Primarily Spanish Learner and Spanish-English Learner groups; thus, those values are not reported separately. Lack of intercorrelations among these measures suggests that each executive function measure was tapping into distinct components of executive function. While these findings are generally expected, one surprise was the lack of relation between the day-night task and the CBQ, since both are purported to sample inhibitory control abilities. Some possible explanations for this null relation are presented in the discussion. 
Table 3 Pearson comelation coefficients (r) among working memory and inhibitory control measures

\begin{tabular}{lcc}
\hline & Day-Night & CBQ IC Index \\
\hline Digit Span $^{\mathrm{a}}$ & .068 & .108 \\
Day-Night &.--032 \\
\hline${ }^{7} 0 e^{*}{ }^{\star} p<.05,{ }^{* \star} p<.01$. &.- \\
${ }^{a}$ Correlations were calculated using raw scores.
\end{tabular}

\section{Language Measures}

Overall performance. Table 4 summarizes performance on measures of receptive vocabulary and language for all participants together, as well as for the Primarily Spanish Learner and Spanish-English Learner groups separately. The table shows that a wide range of language skills were sampled in this study. Standard scores ranged within 2 SDs of the mean on the B-ROWPVT and the Spanish-language SPLS-4, and from greater than 3 SDs below the mean to within 1 SD above the mean on the English-language CELF-P. On the comprehensive Spanish language assessment, all but one child with language delay scored within normal limits (i.e. within 2 SDs of the mean). Scores on the English-language CELF-P were considerably lower, but that is expected given that all kids were learning primarily Spanish. Norms are not available for the test of Spanish grammar, the BESA SMST; however, percent correct scores ranged from very low (12\% correct) to ceiling (100\%). Inspection of performance on the four language measures revealed that all scores were normally distributed in this sample. 
Table 4 Descriptive statistics for language measures

\begin{tabular}{|c|c|c|c|c|c|c|}
\hline & \multicolumn{2}{|c|}{$\begin{array}{l}\text { All participants } \\
(n=36)\end{array}$} & \multicolumn{2}{|c|}{$\begin{array}{l}\text { Primarily Spanish } \\
\text { Learners ( } n=18)\end{array}$} & \multicolumn{2}{|c|}{$\begin{array}{l}\text { Spanish-English } \\
\text { Learners ( } n=18)\end{array}$} \\
\hline & Mean (SD) & Range & Mean (SD) & Range & Mean (SD) & Range \\
\hline \multicolumn{7}{|l|}{ Vocabulary } \\
\hline B-ROWPVT ${ }^{a}$ & $116.2(17.7)$ & $72-145$ & $113.4(18.9)$ & $72-145$ & $118.9(16.5)$ & $89-145$ \\
\hline \multicolumn{7}{|l|}{ Comprehensive Language } \\
\hline SPLS-4 ${ }^{\mathrm{D}}$ (Spanish) & $107.6(15.3)$ & $57-138$ & $109.5(17.6)$ & $57-138$ & $105.7(13.0)$ & $73-125$ \\
\hline CELF-P ${ }^{c}$ (English) & $75.5(16.4)$ & $50-105$ & $71.0(16.0)$ & $50-100$ & $80.0(15.9)$ & $53-105$ \\
\hline \multicolumn{7}{|l|}{ Supplemental Measure } \\
\hline BESA SMST $^{a}$ (Spanish) & $67.6(25.1)$ & $12-100$ & $73.1(23.3)$ & $14-100$ & $62.2(26.4)$ & $12-91$ \\
\hline \multicolumn{7}{|c|}{$\begin{array}{l}\text { Notes: } \\
\text { Vocandard score on the Spanish-English Bilingual Version of the Receptive One-Word Picture } \\
\text { Vocabulary Test (B-ROWPVT) } \\
\text { b Standard score on the Expressive Communication component of the Spanish Preschool } \\
\text { Language Scale, } 4^{\text {th }} \text { Ed. (SPLS-4) } \\
\text { cStandard score on the Expressive Language Scale of the Clinical Evaluation of Language } \\
\text { Fundamentals, Preschool Ed. (CELF-P). Scores should not be interpreted in a standardized } \\
\text { fashion as the population of children tested was not monolingual English-speaking. } \\
\text { dComposite scores are reported, calculated by taking a simple average of percent correct on the } \\
\text { "sentence completion" and "sentence repetition" subsections of the BESA SMST }\end{array}$} \\
\hline
\end{tabular}

Although there was considerable variation in amount of English exposure across participants, the Primarily Spanish Learner and Spanish-English Learner groups did not perform differently from each other on any standardized language measure $(p>0.70)$. Scores on the supplemental measure of Spanish grammar, the BESA SMST, also showed no difference between the two language groups $(p>0.30)$. Note that mean standard score on the CELF-P was considerably lower for all groups, compared to scores of bilingual conceptual vocabulary (B-ROWPVT) and to the Spanish-language SPLS-4. This suggests that although some participants had substantial English language exposure at the time of current testing, English levels were well below that which would be expected for monolingual English learners. This performance is also lower than might be expected for a sample of Spanish-English bilinguals with near-equal 
proficiency in both languages, as in the work of Carlson and Meltzoff (2008). Moreover, the range of scores on the CELF-P was considerable smaller than seen in the other standardized assessments. Thus, not only were the children performing lower as a group on this comprehensive measure of English skill, but also no child was performing at age-equivalent for monolingual peers. This pattern of findings suggests that the process of learning English as a second language was still in early stages for most children.

Intercorrelations among language measures. First-order correlations between the language measures are presented in Table 5 for all participants. As with the executive function measures, similar patterns of intercorrelations were found within the Primarily Spanish Learner and within the Spanish-English Learner groups; thus, those values are not reported separately.

Table 5 Pearson correlation coefficients ( $r$ ) among language measures (full sample $n=36$ )

\begin{tabular}{|c|c|c|c|}
\hline & \multicolumn{2}{|c|}{ Comprehensive Language } & \multirow{2}{*}{$\begin{array}{c}\text { Grammar } \\
\text { BESA SMST } \\
\text { Composite }\end{array}$} \\
\hline & $\begin{array}{c}\text { SPLS-4, } \\
\text { Expressive } \\
\text { Subscale }\end{array}$ & $\begin{array}{l}\text { CELF-P, } \\
\text { Expressive } \\
\text { Lanquage }\end{array}$ & \\
\hline B-ROWPVT & $.553^{\star \star}$ & $.584^{\star \star}$ & $.329^{*}$ \\
\hline $\begin{array}{l}\text { SPLS-4, Expressive } \\
\text { Communication }\end{array}$ & -- & $.433^{\star \star}$ & $.771^{\star \star}$ \\
\hline $\begin{array}{l}\text { CELF-P, Expressive } \\
\text { Language Scale }\end{array}$ & -- & -- & .195 \\
\hline
\end{tabular}
Note: ${ }^{\star} p<0.05,{ }^{\star \star} p<0.01$

In contrast to the executive function measures, there were significant relations among the language measures in this sample. Looking first at the comprehensive language measures, children's performance on the bilingual conceptual vocabulary, B- 
ROWPVT, was positively correlated with performance on both the Spanish-language SPLS-4 and the English-language CELF-P. Moreover, the correlation between the BROWPVT and the SPLS-4 was just as strong as to the English-language CELF-P. In other words, in this sample of preschool children whose first language is Spanish, those children who scored higher on a test of Spanish-English conceptual receptive vocabulary also scored higher on a comprehensive expressive Spanish language test. In addition, children with higher conceptual vocabulary scores were also those who scored higher on a comprehensive expressive English language test. This finding is consistent with strong relations reported among a variety of standardized vocabulary and language tests (Brownell, 2000a; Semel, Wiig, \& Secord, 2004b; Zimmerman, Steiner, \& Pond, 2002a).

Note also that a moderate cross-language correlation was found between the SPLS-4 (expressive Spanish language) and the CELF-P (expressive English language). This is interesting given that the measures test comprehensive knowledge in different languages. However, recall that the B-ROWPVT is highly overlapping with both comprehensive language measures as well. Thus, one possibility is that the relation among all three measures is driven by shared variance. In other words, the SPLS-4 and the CELF-P may be correlated with each other only because of their common relation with the B-ROWPVT. In order to examine this possibility, partial correlations were calculated between the Spanish-language SPLS-4 and the English-language CELF-P, controlling for the B-ROWPVT. This analysis revealed that the SPLS-4 and CELF-P scores were not significantly related once conceptual vocabulary knowledge was accounted for $(p>0.34)$. This suggests that the first-order correlation reported between the SPLS-4 and the CELF-P is driven by those aspects of the tests that are tapping into vocabulary knowledge. Therefore, this could suggest that children who are doing well in 
Spanish beyond the area of vocabulary are not necessarily those children who are doing well in English beyond the area of vocabulary. Understanding this pattern of relations among the vocabulary and language tests will prove useful in the later interpretation of how each of these measures relates to executive function.

Now turning to the supplemental measure of grammar, also listed in Table 5, note that the correlation between the BESA SMST (grammar) and the B-ROWPVT (vocabulary) was also significant, but considerably weaker than those between vocabulary and the comprehensive language assessments. Recall that the BESA SMST includes a sentence repetition task where children must repeat sentences verbatim. Given that repeating unfamiliar words may be more difficult compared to repeating familiar words, it is feasible that reduced vocabulary knowledge could be detrimental to performance on a sentence repetition task. In this way, low scores on the B-ROWPVT could be related to low scores on the BESA SMST. Although grammar and vocabulary are often clinically assessed as two distinct language areas, the significant correlation in this sample further suggests that they may not be completely separable constructs.

The BESA SMST was also positively correlated with the SPLS-4. This may not be surprising given that the BESA SMST and the SPLS-4 are each also correlated with the B-ROWPVT. Similar to the relation between the Spanish-language SPLS-4 and the English-language CELF-P, this could mean that the BESA SMST and the SPLS-4 are not correlated beyond vocabulary knowledge. Again, to examine whether the BESA SMST and the SPLS-4 are related beyond the variance they share with the B-ROWPVT, correlations were examined controlling for the B-ROWPVT. Contrary to the relation between the SPLS-4 and the CELF-P, the Spanish grammar measure (BESA SMST) and the Spanish comprehensive language measure (SPLS-4) remained significantly 
related controlling for vocabulary knowledge $(r(34)=0.749, p<0.001)$. Thus, the relation between the BESA SMST (grammar) and the SPLS-4 (comprehensive language) is not driven by those aspects of each test that are tapping into vocabulary. Children who performed well on Spanish grammar beyond vocabulary are also those children who scored higher on comprehensive expressive Spanish language beyond vocabulary.

Notice finally that no relation was found between the BESA SMST and the CELFP. Thus, children's scores on a measure of Spanish grammar were not related to their expressive English language knowledge. This is consistent with the previous crosslanguage finding that the SPLS-4 and the CELF-P were also not related controlling for receptive vocabulary (B-ROWPVT).

\section{Relations of Language and Executive Function to SES}

Following the work of Carlson and Meltzoff (2008), in this study it was necessary to determine whether socioeconomic status correlated with the language and executive function measures. If so, then any relations found between language and executive function performance might be driven by socioeconomic status alone. In other words, children with higher language scores could also have better working memory abilities simply because they come from a higher socioeconomic environment, where richer language stimulation and/or more experience with executive function tasks may be available. Indeed, the Hollingshead Index was correlated with B-ROWPVT, SPLS-4, CELF-P $(p<0.05$ for all $)$ and digit span $(p<0.001)$. In addition, those children who passed the Standard DCCS task had significantly higher mean HI scores compared to children who failed the task $(p<0.05)$. This suggests that children with higher SES are 
scoring higher on the language measures overall. Children with higher SES also have better working memory and attention-shifting skills.

To summarize the findings thus far, in this study there was a wide range of performance on all language measures, with no group differences between the Primarily Spanish Learners and the Spanish-English Learners. Children, in general, performed better on Spanish language assessments compared to English language assessments. This is not surprising since all children spoke Spanish as their first language and had minimal relative language exposure to English compared to Spanish. Relations among the language measures suggest that vocabulary and comprehensive language measures were related in this sample. Children scoring higher on vocabulary also had higher comprehensive language scores in both languages. In contrast, the EF measures were not related, suggesting that the EF tasks were each tapping into different areas of executive function. Lastly, vocabulary, comprehensive language, verbal working memory and attention-shifting measures were each correlated with SES in this sample.

\section{Relations between Language and Executive Function}

The primary question in this research study asks what the relation is between language measures and tasks that tap into executive function skills. It was expected to find a relation between vocabulary knowledge and working memory and/or inhibitory control, in accordance with Wolfe and Bell $(2004 ; 2007)$. But this study allowed for more detailed exploration of the relation between components of language and components of executive function. By analyzing each EF area separately, it could be determined whether vocabulary, language, or both were related to working memory, inhibitory control, attention-shifting, or a combination of the three executive function areas. If, as 
in previous studies, vocabulary knowledge but not comprehensive language measures are related primarily to working memory, then this would suggest that semantic knowledge in particular is related to verbal working memory. However, if the relations to comprehensive expressive language measures are stronger than those to receptive vocabulary then this may suggest that the link to executive function extends beyond semantic knowledge alone. In order to examine the true relation between language and executive function, partial correlations were computed controlling for SES. Both firstorder and partial correlations are reported in Table 6.

Table 6 First-order and partial correlations among language and executive function measures

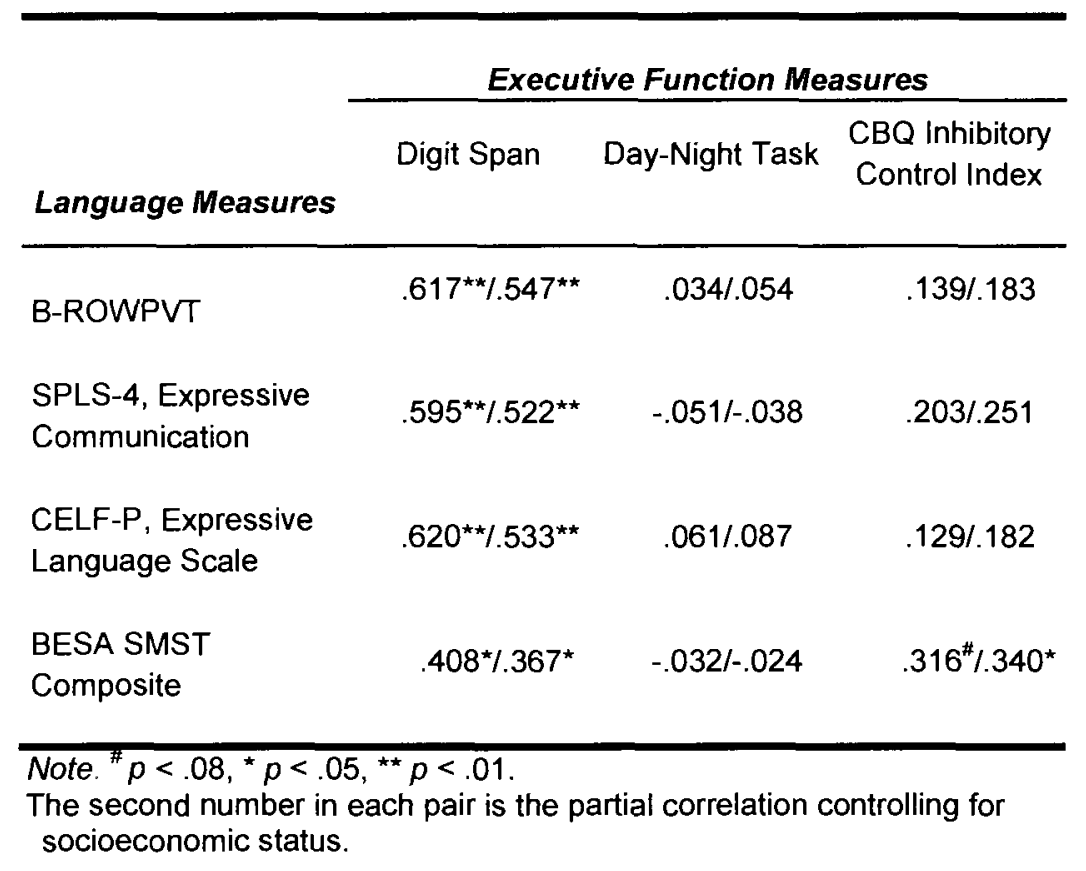

As shown by the first-order correlations in Table 6, all language measures bilingual receptive vocabulary (B-ROWPVT), expressive comprehensive Spanish language (SPLS-4), expressive comprehensive English language (CELF-P), and expressive Spanish grammar (BESA SMST) - were significantly related to verbal working memory performance, as measured by the digit $\operatorname{span}^{3}$. While significant, the 
relation between the BESA SMST and the digit span $(p<0.05)$ was considerably weaker than relations between the digit span and other language measures (all $p<0.005$ ).

Correlations between all language measures and the day-night task were not significant, suggesting that children's scores on tests of language were not related to their abilities to inhibit automatic responses. Similarly, correlations between language measures and the parent-report measure of inhibitory control, the CBQ IC Index, were also not significant. The BESA SMST is one exception to this trend; however, explanations for this finding are not immediately apparent given no known connections in the literature between grammar knowledge and inhibitory control skills. In general, the null relations between language tests and the CBQ IC index are consistent with the null finding between language measures and the laboratory measure of inhibitory control, the daynight task. Some possibilities for these null relations are presented in the discussion. Moving forward with the role of SES in the relations between language and EF, note that the second number in Table 6 shows partial correlations controlling for SES. In all cases, the relation between language and executive function measures remained after controlling for SES. Thus, in this sample, those children with higher language abilities had better working memory, beyond any variance attributable to higher socioeconomic status. The correlation between B-ROWPVT and digit span replicates previous findings that children with higher working memory perform better on tests of receptive vocabulary (Wolfe \& Bell, 2004). Results from the current study show that, in addition to receptive vocabulary, children with better working memory also score higher on comprehensive expressive language measures (SPLS-4 and CELF-P) and on measures of grammar (BESA SMST). The next series of analyses attempts to further 
understand the precise links between performance on the comprehensive battery of vocabulary and language and executive function abilities.

\section{Predictive Relations between Language Skills and Working Memory}

Based on the correlations shown in Table 6, working memory was the executive function component with the strongest relation to language skills in this sample. Recall also from Table 5 that the language measures were highly intercorrelated in this sample. Therefore, several linear regression models were conducted to evaluate the combined

effect $\left(R^{2}\right)$ and independent contributions (unique $r^{2}$ ) of the language predictors. In other words, to what extent does each comprehensive language measure account for variance in working memory over and above the receptive vocabulary measure?

Table 7, Model 1 shows that socioeconomic status (HI score) and receptive vocabulary B-ROWPVT) account for $44.8 \%$ variance in predicting to working memory (digit span) performance. Model 1 shows that $\mathrm{HI}$ score accounts for $6.7 \%$ unique variance, B-ROWPVT accounts for $23.5 \%$ unique variance, and the remaining $14.6 \%$ is from shared variance between socioeconomic status and receptive vocabulary. Note, however, that the B-ROWPVT's unique contribution to working memory is highly significant, even after accounting for socioeconomic status (HI score). 
Table 7 Summary of regression models using language measures as predictors of working memory performance $(n=36)$

\section{Working Memory Performance}

\begin{tabular}{|c|c|c|c|c|}
\hline Lanquage & Model 1 & Model 2 & Model 3 & Model 4 \\
\hline Predictor & Unique $r^{2}$ & Unique $r^{2}$ & Unique $r^{2}$ & Unique $r^{2}$ \\
\hline $\begin{array}{l}\text { Hollingshead } \\
\text { Index }\end{array}$ & $6.7 \%$ * & $2.0 \%, n s$ & $2.0 \%, n s$ & $2.6, n s$ \\
\hline B-ROWPVT & 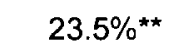 & $2.9 \%, n s$ & $3.0 \%$, ns & $4.9, "$ \\
\hline SPLS-4 & ---- & $5.3 \%{ }^{\#}$ & $1.4, n s$ & -.-- \\
\hline CELF-P & ---- & $5.4 \% "$ & $5.6 \%,{ }^{\#}$ & $7.0 \%$ * \\
\hline $\begin{array}{l}\text { BESA SMST } \\
\text { composite }\end{array}$ & ----- & ----- & $0.2, n s$ & $4.0, n s$ \\
\hline$R^{2}$ & $44.8 \%$ & $56.9 \%$ & $57.0 \%$ & $55.6 \%$ \\
\hline
\end{tabular}

Table 7, Model 2 shows what both comprehensive expressive language measures (SPLS-4 and CELF-P) contribute together and uniquely, over and above SES and receptive vocabulary. First, note that all the variance that the $\mathrm{HI}$ and B-ROWPVT contributed to the working memory prediction was subsumed by the SPLS-4 and the CELF-P. This suggests that when the comprehensive language measures are known, the receptive vocabulary measure is not contributing any additional variance in predicting to working memory. Model 2 shows that adding the comprehensive language measures together accounts for an additional $10 \%$ total variance in the prediction to working memory. The SPLS-4 score adds $6.1 \%$ variance (marginally significant, $p<$ 0.06). Similarly, the CELF-P contributes an additional $5.4 \%$ variance, which is also 
marginally significant $(p<0.06)$. Even when controlling for the other, each comprehensive language measure is making unique contributions to the working memory prediction.

Given that the BESA SMST was included in the current study as a supplemental measure of Spanish grammar knowledge, Model 3 examined the effects of adding the BESA SMST over and above the standardized language measures. First, note that knowing the BESA SMST does not add any significant variance $(p>0.70)$ in the prediction to working memory. Secondly, adding the BESA SMST also subsumes the contributions of the SPLS-4, as indicated by the significant decrease in unique- $r^{2}$ accounted for by the SPLS-4 in Model 3 compared to Model 2. This is not unexpected given that the BESA SMST and the SPLS-4 were highly intercorrelated $(p<0.001)$, even when controlling for vocabulary (B-ROWPVT).

Given the strong relation between the BESA SMST and the SPLS-4, Model 4 examines the working memory prediction using the Spanish grammar measure (BESA SMST) in place of, rather than in addition to, the comprehensive expressive Spanish language measure (SPLS-4). Most notably, the BESA SMST does not contribute significant unique variance. This model shows that the comprehensive expressive Spanish language measure (SPLS-4) is a better working memory predictor compared to a measure of Spanish grammar (BESA SMST).

Lastly, recall that the working memory measure (digit span) was obtained in Spanish for some children and in English for others. The analysis shown in Model 2 was repeated in each of two groups of children, according to language of choice on the digit span. In those children who completed the digit span in Spanish, the SPLS-4 accounted for significant variance $(p<0.05)$, over vocabulary and SES, however, the CELF-P did 
not $(p>0.5)$. Analogously, in those children who completed the digit span in English, it was only the English CELF-P that accounted for significant variance $(p<0.05)$, over and above vocabulary and SES. The Spanish-language SPLS-4 did not $(p>0.1)$. This suggests that language of choice on working memory measures is relevant for predicting working memory performance from language scores in children in early stages of second-language learning.

To summarize findings between language and executive function thus far, data from the current study revealed no relations between vocabulary or comprehensive language and inhibitory control, as measured through a verbal response task (day-night) and a parent-report questionnaire (CBQ). However, there is a robust relation between vocabulary, comprehensive language and Spanish grammar and the measure of verbal working memory, beyond that attributable to socioeconomic status. Moreover, regression analyses revealed that comprehensive language measures do indeed account for variance over and above the receptive vocabulary measure alone.

\section{Relations between Language and Attention-Shifting}

Attention-shifting, also known as "shifting mental set" requires an individual to selectively attend to a different dimension of a stimulus in such a way the new response set is in conflict with a previous response rule. In the current study, the Standard DCCS was used to assess attention-shifting. Recall that the main dependent measure in the Standard DCCS task was a binary pass or fail outcome. That is, children were either able or not able to shift their "mental set." To explore relations between scores on language tests and this pass-fail measure, Figures 4 and 5 show mean standard scores on the B-ROWPVT, SPLS-4, and CELF-P for those children who passed the Standard DCCS compared to those who failed the task. All participants are examined first (Figure 
4), and then children in the two subgroups: the Primarily Spanish Learners (Figure 5a), and the Spanish-English Learners (Figure 5b) are examined.

Looking first at all participants (Figure 4), performance on the vocabulary

measure (B-ROWPVT) was in the hypothesized direction, but only marginally significant. Note, however, that mean scores on both comprehensive expressive language measures (the SPLS-4 and the CELF-P) were significantly higher for children who passed the Standard DCCS task. This suggests that children who demonstrated the ability to effectively shift their attention in this task, scored higher on all language tests compared to those children who were not able to shift their attention.

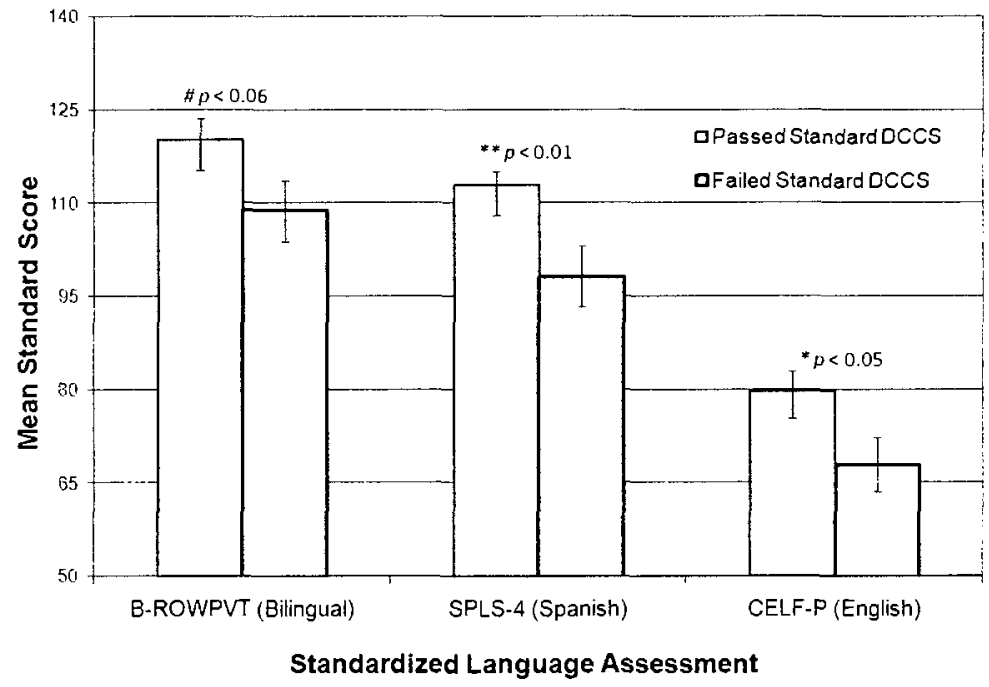

Figure 4 Mean standard score on language assessments according to pass versus fail outcome on the Standard DCCS task for all participants $(n=36)$. Bars represent standard errors. 

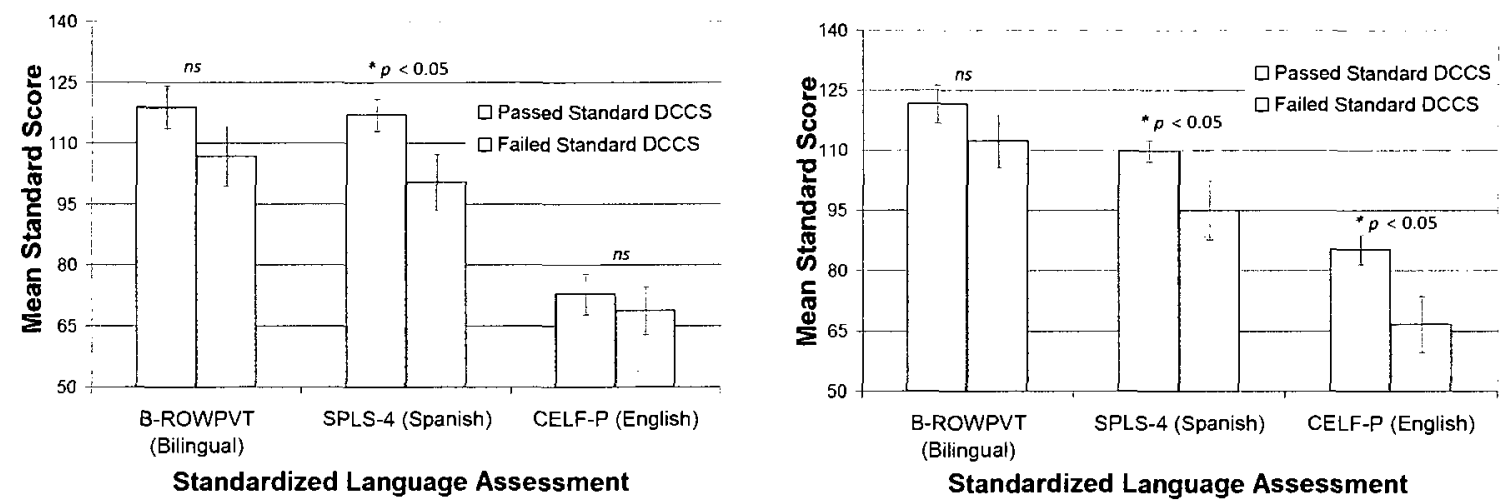

Figure 5a \& b Mean standard score on language assessments according to pass versus fail outcome on the Standard DCCS task for (a) the Primarily Spanish Learners $(n=18)$ and (b) the Spanish-English Learners $(n=18)$. Bars represent standard errors.

In both groups of language learners (Figures $5 a$ and $5 b$ ), the children who passed the DCCS task scored generally higher on receptive vocabulary (B-ROWPVT); however, in both groups of children, the difference was not statistically reliable. In contrast, on the comprehensive expressive Spanish language measure (SPLS-4), both Primarily Spanish Learners and Spanish-English Learners who passed the Standard DCCS scored higher than their peers who failed the attention-shifting task. The main difference between the language and attention-shifting relation, however, is seen in the English CELF-P. Primarily Spanish learners did equally poorly on the English CELF-P whether or not they passed the Standard DCCS. It is not surprising that attentionshifting performance did not differentiate performance on the English CELF-P for those children not learning much English. However, Spanish-English learners who passed the Standard DCCS performed significantly higher on the CELF-P compared to their peers who failed the task.

Lastly, the measure of grammar is examined. The final analysis compared mean BESA composite score for those children who passed the attention-shifting task (Standard DCCS) compared to those who failed it. Note that the left panel of Figure 6 
shows mean BESA composite scores for all participants, whereas the middle panel shows mean scores for the Primarily Spanish Learners, and the right panel shows the Spanish-English Learners.

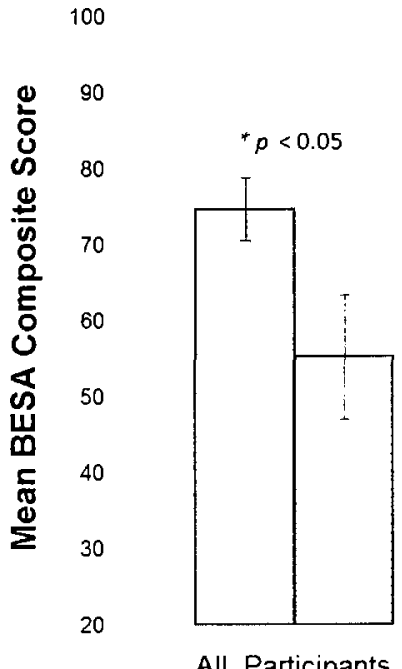



Primarily Spanish

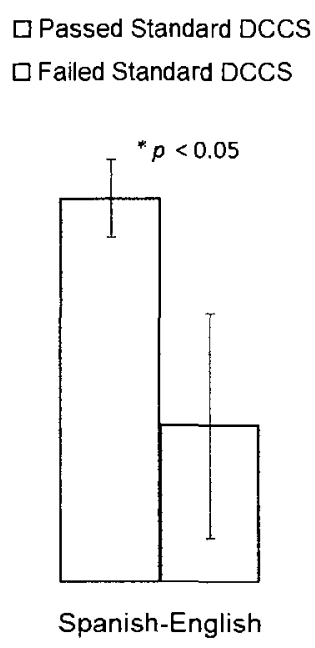

Participant Groups

Figure 6 Mean composite scores on the BESA SMST according to pass versus fail outcome on the Standard DCCS task. Bars represent standard errors.

Consistent with findings from the standardized measures, children who passed the Standard DCCS performed better on Spanish grammar compared to children who failed. This finding was particularly robust in the sample as a whole and in the SpanishEnglish learners. While in the right direction, this difference was not statistically reliable in the Primarily Spanish Learners. One possibility is that the small sample size of Primarily Spanish Learners passing versus failing the Standard DCCS task may have accounted for the null result in this language group compared to the Spanish-English Learners. 
To summarize findings between attention-shifting and language measures, group differences for the children who pass versus fail the Standard DCCS task are consistently better for the comprehensive language measures than they are for vocabulary or grammar alone.

\section{Summary of Findings}

The current study replicates previous findings linking language measures and executive function performance at the preschool age (McClelland et al., 2007; Wolfe \& Bell, 2004; Wolfe \& Bell, 2007). It is particularly interesting that these results were obtained in a sample of Spanish-speaking preschoolers in the United States, given that there is little research on EF development in this population. Importantly, relations of language to EF performance were not equal across all measures. Language relations were strongest to the EF areas of working memory and attention-shifting. Moreover, the current study extended previous work by showing that language measures beyond receptive vocabulary were powerful predictors of concurrent EF ability.

\section{Discussion}

This study extended previous work examining relations between language and executive function in four unique ways. First, executive function areas were explored more broadly by analyzing the three areas of working memory, inhibitory control, and attention-shifting individually in relation to language measures. Second, by focusing on low SES Spanish speakers, research was extended to an understudied population in terms of both language and socioeconomic background. Third, because some participants had multiple language exposure, this study allowed for the exploration of hypotheses concerning EF performance in bilingual individuals. Finally, this study 
extended language measures beyond the isolated construct of receptive vocabulary to include comprehensive expressive language measures.

\section{Examining Executive Function Relations to SES in Spanish Speakers}

The first two goals of this study expanded current knowledge of executive function through the administration of multiple EF measures to a sample of low SES Spanish speakers. In this way the findings contributed to an understanding of EF in a broader population compared to earlier studies of middle and upper middle class English speakers. The three constructs of working memory, inhibitory control, and attentionshifting were selected as the executive function areas of focus in this study. Relations between language and working memory were predicted, as have been documented in previous work. Relations to inhibitory control, however, were not necessarily expected given that previous studies have reported language relations to composite scores of working memory, inhibitory control, and attention-shifting. However, EF was analyzed as a composite score, rather than examining each construct separately. Thus, it is possible that one construct was driving the relation in those studies and that not all EF areas have a relation to language. In addition, in this study relations of both language and executive function to SES were closely analyzed, even in this sample restricted primarily to the low end of the SES continuum.

Looking first at inhibitory control, participants in this study performed similarly to those participants from middle to upper-middle class monolingual English-speaking peers, as reported in the original Gerstadt et al. (1994) paper. In other words, absolute performance on the day-night task was comparable in this low SES sample and in high SES samples reported in the existing research literature. Further, no relation was found between day-night performance and SES in the predominantly low SES group sampled 
in this study. Taken together the lack of relations to SES suggest that this measure may be appropriate for use with children from other ethnic and socioeconomic backgrounds. Nevertheless, future studies examining the day-night task in children from other cultural and social backgrounds will further inform the cross-cultural appropriateness of this EF measure.

In contrast to the day-night task, performance on the digit span task was greater than one standard deviation below the mean for these children compared to norms for monolingual English-speaking peers. This suggests that the sample of children in this study may not be as adept at repeating sequences of numbers compared to their middle class English-speaking peers. Recall also that digit span performance correlated with SES, even in this restricted sample of predominantly low SES participants. There was an absolute difference in working memory capacity between middle class monolingual English speakers and the children in this study as well as a within-group relation between working memory and SES in this low SES sample. Together, these findings suggest that children's working memory capacity is likely to be impacted by those experiences that are correlated with socioeconomic status. Future research directly comparing working memory performance in low versus high SES children from the same cultural and linguistic background would provide more detailed information about the relations between working memory and SES than this study was able to provide.

This study was also the first to use a Spanish adaption of the Standard Dimensional Change Card Sort task as a measure of attention-shifting in Spanish speakers. Although the Standard DCCS task has been used to test bilinguals (Bialystok, 1999), no known studies have administered the Standard DCCS in a language other than English. In addition, the participants in the current study were from a lower SES 
sample than has been previously tested on the Standard DCCS task. Recall that based on studies of higher SES monolingual English-speaking populations, one would expect most children in the current study to pass the Standard DCCS task. Indeed, some children were quite successful at the DCCS task. However, the overall passing rate of $63.9 \%$ was considerably lower compared to other studies of older 4 -year-olds. The rate in this study was more comparable to the passing rate reported for younger as opposed to older 4-year-olds. Recall, however that pass-fail outcome in the current study was also related to SES. In this sample, children who passed the DCCS tended to be higher SES compared to children who failed the DCCS task. Thus, it is possible that overall passing rates in the current sample were slightly depressed due to the lower SES population.

Several possibilities account for lower working memory and attention-shifting performance in this sample of children. It is possible that children from lower SES backgrounds may not have language experiences that exploit EF in the same way as higher SES peers. Higher SES children may partake in more daily activities that tax working memory and attention skills. All but a few children in current study attended some form of preschool. While the preschool experience was varied across children in the current study, detailed information about the types of activities children completed in preschool was not available. Future research should explore daily activities of these children in more detail to see if variation in daily experiences with tasks taxing executive function abilities account for differences in performance on EF measures.

The SES differences in this sample may also explain why working memory and attention-shifting were more difficult for the children in the current study compared to that reported in the literature for higher SES peers. Children in this population may have 
performed less well on the digit span task because they have less experience with math skills or number play compared to peers from American, English-speaking homes or to higher SES peers from Spanish-speaking backgrounds. Inhibitory control, however, was not as taxing. One explanation for this may be that the day-night inhibitory control task does not require manipulation of verbal material compared to the digit span task. Therefore, the verbal component of the digit span may be tapping into a particularly vulnerable skill in this population. Regarding differences in attention-shifting skill, the Standard DCCS task can be described as having both an inhibitory control component (i.e., resisting the urge to sort by the first dimension) and a working memory component (i.e., remembering which sorting rule to apply). In contrast, the day-night task may only tap into inhibitory control. In this way, the Standard DCCS may be a fundamentally harder task than the day-night.

Results showed that SES is indeed related to executive function, particularly to working memory and attention-shifting. Recall that while the sample of children in this study was predominantly low SES, in general children had age-level Spanish-language skills. Spanish language performance was representative of the larger population, in terms of both range and mean scores. Further, receptive vocabulary and comprehensive expressive language in both Spanish and English were each correlated to SES in this sample as well, as has been documented with English-speaking samples in the research literature. Future studies should explore why SES is also related to executive function not only in middle-class English-speaking children but also in broader populations such as the one in this study. 


\section{Examining EF Measures Individually rather than as a Composite Score}

Previous studies examining links between language and EF have used either a single $\mathrm{EF}$ task or $\mathrm{EF}$ composite scores to correlate EF skills to language ability (Wolfe \& Bell, 2004; Wolfe \& Bell, 2007). This study analyzed the three EF areas of working memory, inhibitory control, and attention-shifting separately in order to understand whether all or only some EF areas are related to language. The pattern of nonsignificant intercorrelations in this study revealed that each EF task measured different EF skills. This was a desirable finding given that the aim was to administer tasks sampling different EF areas, with the ultimate goal of disentangling which EF skills were more intimately linked to language.

On the other hand one pattern of relations was unexpected in this study. The null relation between the day-night and the CBQ IC index was surprising given that both were collected as measures of inhibitory control ability. This finding differs from other studies reporting relations between CBQ IC and performance on the delay of gratification ("hot" inhibitory control) and Go-No Go ("cold" inhibitory control) tasks (Davis, Bruce, \& Gunnar, 2002). Also, it should be noted that Wolfe and Bell (2007) found a significant relation between the CBQ IC index and the yes-no task ("cold" inhibitory control). Importantly, however, they failed to find a correlation between the CBQ IC index and the day-night task in a sample of $4 \frac{1}{2}$-year-old children. It may be that only one of the inhibitory control measures in the current study accurately captured inhibitory control ability. Either the day-night task was a poor measure of inhibitory control or the parent-report measure (CBQ) was inappropriate for sampling inhibitory control behavior in this sample. 
The first possibility is that the day-night task did not adequately tap into inhibitory control the way it was administered in this study. More specifically, the abbreviated daynight task used in the current study may not have had enough testing trials to accurately measure inhibitory control ability in this population. However, absolute performance on the abbreviated task was comparable to that reported for 4-year-olds in the original daynight study (Gerstadt et al., 1994). Thus, it is not likely that the shortened form of the task accounts for the low correlation between day-night and CBQ IC index.

An alternative explanation for the null relation between day-night and the CBQ IC index is that the CBQ questionnaire yielded unreliable data from the current sample. Parents in this sample may have had different perceptions or cultural expectations of inhibitory control behavior in their children compared to parents of middle-class American children. It could also be that items in the questionnaire tapped into middleclass American cultural routines or activities unfamiliar to the families in this study. The official Castilian Spanish translation of the CBQ was used in this study, with minor changes in wording made throughout to account for vocabulary differences in Mexican Spanish. It was noted, however, that parents were required to comment on their children's behavior in some situations that may have been culturally unfamiliar (e.g., while playing "Simon says").

Still a third possibility is that the vocabulary or sentence structure used in the Spanish CBQ was at an academic level which may have been unfamiliar to some parents, in particular those with low education. Some parents may have had difficulty completing the questionnaire independently and may have contributed unreliable data as a result. Further studies should explore parenting beliefs and values on the CBQ in other language and cultural groups, taking into account external factors such as literacy 
and education levels when administering such questionnaires. In summary, the EF measures in this study were not intercorrelated with each other, suggesting that research on EF skills should select a variety of EF constructs and in turn analyze each EF skill separately rather than as EF composite scores.

\section{Performance in Primarily Spanish versus Spanish-English Learners}

An important question in the EF literature is the extent to which bilingual exposure impacts EF skills. Recall that the current study tested participants from a larger longitudinal study and that some children were exposed to relatively more English than other children. Thus, a third goal of this study was to examine the performance of Spanish-English learners compared to primarily monolingual Spanish learners. At the outset of this study, it was expected that some children would have considerably more English exposure, even though they began participation in the larger longitudinal study at 18 months as monolingual Spanish-learning children. Given expected betweensubject variation in exposure to English at the time of participation in the current study, language exposure was documented for each participant. It was found that there were indeed some children who could be considered Spanish-English Learners. Therefore, in the current study children were categorized into Primarily-Spanish versus SpanishEnglish Learners. However, the Spanish-English Learners did not perform significantly different from the Primarily Spanish Learners on any EF task.

While this may seem surprising given the "bilingual advantage" in the EF literature, several reasons account for this finding in the current sample tested. Foremost, a closer look at language scores in this sample revealed that even those children who were grouped as Spanish-English Learners were not truly bilingual. As a group, the Spanish-English Learners were not equally proficient in $L 1$ and $L 2$, as were 
the bilinguals in Bialystok (1999) and Carlson and Meltzoff (2008). Instead, SpanishEnglish Learners were most likely at the beginning stages of $L 2$ acquisition. In this way, they were more similar to the group of early second-language learners reported in Carlson and Meltzoff (2008) who also did not show a bilingual advantage compared to the group of balanced bilingual children.

Factors beside the bilingual status of the Spanish-English Learners also may account for the lack of a "bilingual advantage" in EF tasks. For example, it is possible that the small sample size of 18 children in each language group did not provide enough statistical power to reveal a difference in performance on EF tasks at the group level. Further, a larger bilingual sample would also likely provide more range in proportion of Spanish-English language exposure in the Spanish-English Learner group. In this study, even the Spanish-English Learners (i.e., the children identified as the more bilingual children of the group) had significantly greater Spanish exposure compared to English. It is possible that a bilingual group with more varied language exposure may show some differences in EF tasks. While the results of the current study did not find any evidence of the "bilingual advantage," ongoing studies are currently exploring EF skills in children who are simultaneous bilinguals, acquiring both Spanish and English from an early age.

\section{Exploring Language beyond the Construct of Receptive Vocabulary}

The primary goal of the current study was to explore relations between executive functioning skills and language using several measures. Results indicated that language skills were not related to all EF constructs. Results showed that language measures were consistently predictive of working memory and were significantly higher for children with better attention-shifting skills, whereas no consistent relations were found between language and the measures of inhibitory control. Future studies of EF skills in children 
and their relation to other cognitive or language skills should analyze EF areas individually rather than relying on composite scores.

In examining specific language to EF relations, the verbal working memory measure was found to be most robustly related to language. Perhaps this is not surprising given that the digit span was the EF task which most heavily relied on verbal manipulation of information. However, recall also that children who passed the attention-shifting task were more likely to have significantly higher scores on both comprehensive expressive language measures but not on receptive vocabulary. Again comprehensive language measures captured language skill beyond vocabulary, and in doing so revealed the language relation to attention-shifting.

In contrast to the working memory measure which involved manipulation of verbal information, the relation between attention-shifting and language is less straightforward and has not been previously documented in the literature. One possibility is that skilled language learners in this sample used strategies, such as selftalk, to regulate attention better than their peers with weaker language abilities. In the Standard DCCS task, children were verbally given cues containing with the information about which of the two dimensions was relevant for sorting the stimulus cards (i.e., during color-sorting trials, children heard "here's a blue one" while during animal-sorting trials children heard "here's a cat."). It is possible that children with better language skills were more adept at using this verbal information as an external cue to direct their attention to the relevant dimension (Munakata, Morton, \& Yerys, 2003). It is also possible that success on Standard DCCS task may be linked to success in language more generally because children who are able to pull their attention across different contexts may be better at extracting relations from the language input they hear. 
Lastly, while all language measures were related to working memory and attention-shifting, the comprehensive measures were more robustly related than receptive vocabulary or expressive grammar alone. Previous studies linking language performance to EF skills only examine receptive vocabulary, yet they make claims about relations to overall language. In using receptive vocabulary measures, researchers only captured an individual's semantic knowledge. No information was obtained about the numerous other skills (e.g., morphology, syntax) required for understanding and producing language.

By collecting comprehensive language measures in this study, a more complete picture of each child's language competence was obtained. Further, it was found that those language areas beyond semantic knowledge contributed significant unique variance to the concurrent EF prediction. Therefore, not only was more accurate information obtained about children's language skills, but also this more comprehensive language information accounted for more variability in EF performance.

\section{Limitations of the Current Study}

Although the current study extended the EF literature in significant ways, there are several limitations to the results obtained by this study. First, socioeconomic status of the participants was limited to the lower end of the SES continuum, as opposed to sampling the full range of low to high SES participants. Despite the predominantly low SES sample, relations of SES to language and EF were significant, suggesting that SES affects language and EF in robust ways, and must therefore be controlled for in studies of language and EF. Secondly, the current study focused on a single age group, preschool-aged children. As a cross-sectional study, there was no information about development of EF abilities in individual children prior to the current testing age. 
Additionally, this study cannot offer data on the predictive validity of specific EF tasks to later language or EF outcomes.

A second limitation of the current research is that while comprehensive measures account for more variance, it is unclear what facet of comprehensive language beyond semantics contributed significant additional variance in predicting to EF. One possibility is that expressive measures account for more variance. Inclusion of a separate expressive vocabulary measure in addition to receptive vocabulary and comprehensive expressive language would permit analyses to answer this question. Lastly, caution should be used when generalizing to other populations. The results were obtained with a low SES sample of Spanish-speaking preschoolers. However, caution need apply when generalizing the current results to other language, SES, or age groups.

\section{Future Research}

The interest in language and EF relations emerged largely out of clinical observations of co-occurring language and EF problems. Future research should connect the current findings with the clinical literature. One way to extend the current research is to test a sample of children with $S L I$ on the batteries of language and EF tasks used in this study, and to compare relations between language and EF in a sample

of children with language disorders to a sample of typically-developing children. There is some evidence that children with SLI may be qualitatively different from typicallydeveloping peers with respect to working memory and language abilities (Ellis Weismer et al., 1999), and this possibility merits further study using broader language and EF measures.

A second clinical application of the current results is exploring whether strengthening executive function skills results in improvements in language performance. 
A recent EF intervention study in low-income preschoolers at risk for later academic problems revealed significant improvements in cognitive control following training in the EF areas of inhibitory control, working memory, and attention-shifting (Diamond, Barnett, Thomas, \& Munro, 2007). If EF training also resulted in improved language skills, delivery of special education services, including speech and language, could be significantly impacted. In particular, such work could directly affect therapy for children with co-morbid cognitive and language impairments.

\section{Conclusions}

The first major finding from the current study is that that EF performance should be analyzed according to individual areas. Analyzing EF performance via composite scores may be sufficient for obtaining an overall picture of EF relations to other linguistic and non-linguistic skills. However, such composite scores can also mask more specific relations to particular executive function areas.

Secondly, this study extended EF research to a historically underserved and understudied population. It is the first to provide data on Spanish versions of the daynight and Standard DCCS tasks in an effort to extend use of these EF measures to other cultural and linguistic groups of interest. In extending these measures to a different population, the importance of controlling for socioeconomic status was underscored, particularly since SES was strongly related to both language skills and EF development even in this limited sample of predominantly low SES participants.

Finally, the third, and most important, point is that language measures are robustly related to working memory and attention-shifting. Comprehensive measures are better predictors of executive function compared to receptive vocabulary alone. From a clinical standpoint, comprehensive language measures are better for assessing 
a child's full repertoire of language abilities across multiple language areas. This study shows that comprehensive language measures are also more powerful predictors of EF performance compared to isolated measures of language. 


\section{References}

American Psychiatric Association. (2000). Diagnostic and statistical manual of mental disorders (4th ed.). Washington, DC: Author.

Anderson, V., Anderson, P. J., Jacobs, R., \& Smith, M. S. (2008). Development and assessment of executive function: From preschool to adolescence. In $\mathrm{V}$. Anderson, R. Jacobs, \& P. J. Anderson (Eds.), Executive functions and the frontal lobes: A lifespan perspective (pp. 123-154). Philadelphia, PA: Taylor \& Francis.

Ardila, A., Rosselli, M., Matute, E., \& Guajardo, S. (2005). The influence of the parents' educational level on the development of executive functions. Developmental Neuropsychology, 28, 539-560.

Arriaga, R. K., Fenson, L., Cronan, T., \& Pethick, S. J. (1998). Scores on the MacArthur Communicative Development Inventory of children from low- and middle-income families. Applied Psycholinguistics, 19, 209-225.

Baddeley, A. (1996). The concept of working memory. In S. Gathercole (Ed.), Models of short-term memory (pp. 1-27). Hove, UK: Psychology Press.

Banich, M. (2009). Executive function: The search for an integrated account. Current Directions in Psychological Science, 18, 89-94.

Bavin, E., Wilson, P., Maruff, P., \& Sleeman, F. (2005). Spatio-visual memory of children with Specific Language Impairment: Evidence for generalized processing problems. International Journal of Language and Communication Disorders, 40 , 319-332.

Bedore, L., \& Peña, E. (2008). Assessment of bilingual children for identification of language impairment: Current findings and implications for practice. International Journal of Bilingual Education and Bilingualism, 11, 1-29.

Bialystok, E. (1997). Effects of bilingualism and biliteracy on children's emerging concepts of print. Developmental Psychology, 33, 429.

Bialystok, E. (1999). Cognitive complexity and attentional control in the bilingual mind. Child Development, 70, 636-644. 
Brindis, C. D., Driscoll, A. K., Biggs, M. A., \& Valderrama, L. T. (2002). Fact sheet on Latino youth : Income \& poverty. University of California, San Francisco, Center for Reproductive Health Research and Policy, Department of Obstetrics, Gynecology and Reproductive Health Sciences and the Institute for Health Policy Studies, San Francisco, CA.

Brownell, R. (2000a). Receptive One-Word Picture Vocabulary Test manual. Novato, CA: Academic Therapy Publications, Inc.

Brownell, R. (2000b). Receptive One-Word Picture Vocabulary Test: Spanish-Bilingual Edition. Novato, CA: Academic Therapy Publications, Inc.

Carlson, S.M. (2005). Developmentally sensitive measures of executive function in preschool children. Developmental Neuropsychology, 28, 595-616.

Carlson, S. M., \& Meltzoff, A. N. (2008). Bilingual experience and executive functioning in young children. Developmental Science, 11, 282-298.

Davis, E. P., Bruce, J., \& Gunnar, M. R. (2002). The anterior attention network: Associations with temperament and neuroendocrine activity in 6-year-old children. Developmental Psychobiology, 40, 43-56.

Dawson, G., Meltzoff, A., Osterling, J., \& Rinaldi, J. (1998). Neuropsychological correlates of early symptoms of autism. Child Development, 69, 1276-1285.

Diamond, A. (1985). Development of the ability to use recall to guide action, as indicated by infants' performance on AB. Child Development, 56, 868-883.

Diamond, A. (2006). The Early Development of Executive Functions. In E. Bialystok, \& F. I. M. Craik (Eds.), Lifespan cognition: Mechanisms of change (pp. 70-95). New York, NY US: Oxford University Press.

Diamond, A., Barnett, W., Thomas, J., \& Munro, S. (2007). Preschool program improves cognitive control. Science, 318, 1387-1388.

Dollaghan, C., \& Campbell, T. (1998). Nonword repetition and child language impairment. Journal of Speech, Language, and Hearing Research, 41, 11361146. 
Dollaghan, C. A., Campbell, T. F., Paradise, J. L., Feldman, H. M., Janosky, J. E., Pitcairn, D. N., et al. (1999). Maternal education and measures of early speech and language. Journal of Speech, Language, and Hearing Research, 42, 14321443.

Dunn, L. M., \& Dunn, L. M. (1997). Peabody Picture Vocabulary Test-Third Edition. Circle Pines, MN: American Guidance Service.

Dunn, L. M., Lugo, D. E., Padilla, E. R., \& Dunn, L. M. (1986). Test de Vocabulario en Imágenes Peabody: Adaptación Hispanoamericana. Circle Pines, MN: American Guidance Service.

Ellis Weismer, S., Evans, J., \& Hesketh, L. (1999). An examination of verbal working memory capacity in children with specific language impairment. Journal of Speech, Language, and Hearing Research, 42, 1249-1260.

Fenson, L., Marchman, V. A., Thal, D., Dale, P. S., Reznick, J. S., \& Bates, E. (2007). MacArthur-Bates Communicative Development Inventories : User's Guide and Technical Manual, Second Edition. Baltimore, MD: Brookes Publishing Co.

Fernald, A., Zangl, R., Portillo, A. L., \& Marchman, V. A. (2008). Looking while listening: Using eye movements to monitor spoken language comprehension by infants and young children. In I. Sekerina, E. M. Fernández, \& H. Clahsen (Eds.), Developmental psycholinguistics: On-line methods in children's language processing (pp. 97-135). Amsterdam: John Benjamins.

Frye, D., Zelazo, P., \& Palfai, T. (1995). Theory of mind and rule-based reasoning. Cognitive Development, 10, 483-527.

Garon, N., Bryson, S., \& Smith, I. (2008). Executive function in preschoolers: A review using an integrative framework. Psychological Bulletin, 134, 31-60.

Gathercole, S., Service, E., Hitch, G., Adams, A., \& Martin, A. (1999). Phonological short-term memory and vocabulary development: Further evidence on the nature of the relationship. Applied Cognitive Psychology, 13, 65-77.

Gerstadt, C., Hong, Y., \& Diamond, A. (1994). The relationship between cognition and action: Performance of children $31 / 2-7$ years old on a Stroop-like day-night test. Cognition, 53, 129-153. 
Golden, C. (2002). Stroop Color and Word Test. Lutz, FL: Psychological Assessment Resources.

Grant, D. A., \& Berg, E. A. (1993). Wisconsin Card Sorting Test. Lutz, FL: Psychological Assessment Resources.

Griffith, E. M., Pennington, B. F., Wehner, E. A., \& Rogers, S. J. (1999). Executive functions in young children with autism. Child Development, 70, 817-832.

Gutiérrez-Clellen, V., Restrepo, M., \& Simón-Cereijido, G. (2006). Evaluating the discriminant accuracy of a grammatical measure with Spanish-speaking children. Journal of Speech, Language, and Hearing Research, 49, 1209-1223.

Guttentag, R.E., Haith, M.M., Goodman, G.S., \& Hauch, J. (1984). Semantic processing of unattended words in bilinguals: A test of the input switch mechanism. Journal of Verbal Learning and Verbal Behavior, 23, 178-188.

Hollingshead, A.B. (1975). Four factor index of social status. Unpublished manuscript, Yale University, New Haven, CT.

Hughes, C., \& Ensor, R. (2005). Executive function and theory of mind in 2 year olds: A family affair? Developmental Neuropsychology, 28, 645-668.

Joseph, R. M., McGrath, L. M., \& Tager-Flusberg, H. (2005). Executive dysfunction and its relation to language ability in verbal school-age children with autism. Developmental Neuropsychology, 27, 361-378.

Jurado, M., \& Rosselli, M. (2007). The elusive nature of executive functions: A review of our current understanding. Neuropsychology Review, 17, 213-233.

Just, M., \& Carpenter, P. (1992). A capacity theory of comprehension: Individual differences in working memory. Psychological Review, 99, 122-149.

Kirkham, N., Cruess, L., \& Diamond, A. (2003). Helping children apply their knowledge to their behavior on a dimension-switching task. Developmental Science, 6, 449476. 
Langdon, H. (2008). Assessment and intervention for communication disorders in culturally and linguistically diverse populations. United States: Thomson Delmar Learning.

Leon-Carrion, J., Garcia-Orza, J., \& Pérez-Santamaria, F. (2004). Development of the inhibitory component of the executive functions in children and adolescents. International Journal of Neuroscience, 114, 1291-1311.

Leonard, L. B. (1998). Children with specific language impairment. Boston: The MIT Press.

Logan, G., \& Cowan, W. (1984). On the ability to inhibit thought and action: A theory of an act of control. Psychological Review, 91, 295-327.

Marchman, V.M., Martínez-Sussmann, C., \& Dale, P. (2004). The language-specific nature of grammatical development: Evidence from bilingual language learners. Developmental Science, 7, 212-224.

Martin, N. (2005). Test of Auditory Processing Skills, Third Edition, Spanish Bilingual Edition. Novato, CA: Academic Therapy Publications.

Martin, N., \& Brownell, R. (2005). Test of Auditory Processing Skills, Third Edition. Novato, CA: Academic Therapy Publications.

Marton, K., Kelmenson, L., \& Pinkhasova, M. (2007). Inhibition control and working memory capacity in children with SLI. Psychologia, 50, 110-121.

Matute, E., Rosselli, M., Ardila, A., \& Morales, G. (2004). Verbal and nonverbal fluency in Spanish-speaking children. Developmental Neuropsychology, 26, 647-660.

McCarthy, D. (1970). McCarthy Scales of Children's Abilities. New York: Psychological Corporation.

McClelland, M. M., Cameron, C. E., Connor, C. M., Farris, C.L., Jewkes, A. M., \& Morrison, F. J. (2007). Links between behavioral regulation and preschoolers' literacy, vocabulary, and math skills. Developmental Psychology, 43, 947-959. 
Miyake, A., Friedman, N. P., Emerson, M. J., Witzki, A. H., Howerter, A., \& Wager, T. D. (2000). The unity and diversity of executive functions and their contributions to complex "frontal lobe" tasks: A latent variable analysis. Cognitive Psychology, 41, 49-100.

Montgomery, J. (1995). Sentence comprehension in children with specific language impairment: The role of phonological working memory. Journal of Speech and Hearing Research, 38, 187-199.

Montgomery, J. (2003). Working memory and comprehension in children with specific language impairment: What we know so far. Journal of Communication Disorders, 36, 221.

Morton, J., \& Harper, S. (2007). What did Simon say? Revisiting the bilingual advantage. Developmental Science, 10, 719-726.

Munakata, Y., Morton, J., \& Yerys, B. (2003). Children's perseveration: attentional inertia and alternative accounts. Developmental Science, 6, 471-473.

Mundy, P., Delgado, C., Block, J., Venezia, M., Hogan, A., \& Seibert, J. (2003). A Manual for the Abridged Early Social Communication Scales (ESCS). Unpublished manual, University of Miami.

Obonsawin, M. C., Crawford, J. R., Page, J., Chalmers, P., Cochrane, R., \& Low, G. (2002). Performance on tests of frontal lobe function reflect general intellectual ability. Neuropsychologia, 40, 970-977.

Oram, J., Fine, J., Okamoto, C., \& Tannock, R. (1999). Assessing the language of children with attention deficit hyperactivity disorder. American Journal of SpeechLanguage Pathology, 8, 72-80.

Peña, E. D., Gutiérrez-Clellen, V. F., Iglesias, A., Goldstein, B., \& Bedore, L. M. (2007). Bilingual English Spanish assessment. Manuscript in preparation.

Putnam, S. P., \& Rothbart, M. K. (2006). Development of short and very short forms of the Children's Behavior Questionnaire. Journal of Personality Assessment, 87, 103-113. 
Riccio, C., Cash, D., \& Cohen, M. (2007). Learning and memory performance of children with specific language impairment (SLI). Applied Neuropsychology, 14, 255-261.

Roid, G., \& Miller, L. (1997). Leiter International Performance Scale-Revised. Los Angeles, CA: Western Psychological Services.

Rothbart, M. K., Ahadi, S. A., Hershey, K. L., \& Fisher, P. (2001). Investigations of temperament at 3-7 years: The Children's Behavior Questionnaire. Child Development, 72, 1394-1408.

Saffran, J., Aslin, R., \& Newport, E. (1996). Statistical learning by 8-month-old infants. Science, 274, 1926-1928.

Salthouse, T. (1996). The processing-speed theory of adult age differences in cognition. Psychological Review, 103, 403-428.

Semel, E., Wiig, E. H., \& Secord, W. (2004a). Clinical Evaluation of Language Fundamentals - Preschool (2nd ed.). San Antonio, TX: The Psychological Corporation.

Semel, E., Wiig, E. H., \& Secord, W. (2004b). Clinical Evaluation of Language Fundamentals - Preschool (2nd ed.): Examiner's manual. San Antonio, TX: The Psychological Corporation.

Sohlberg, M. M., \& Mateer, C.A. (2001). Cognitive Rehabilitation: An Integrative Neuropsychological Approach. New York: The Guilford Press.

Spaulding, T., Plante, E., \& Vance, R. (2008). Sustained selective attention skills of preschool children with Specific Language Impairment: Evidence for separate attentional capacities. Journal of Speech, Language, and Hearing Research, 51, 16-34.

Tomasello, M., \& Cale Kruger, A. (1992). Joint attention on actions: Acquiring verbs in ostensive and non-ostensive contexts. Journal of Child Language, 19, 311-333.

US Census Bureau (2000). Retrieved October 18, 2009, from http://www.census.gov. 
Wolfe, C. D., \& Bell, M. A. (2004). Working memory and inhibitory control in early childhood: Contributions from physiology, temperament, and language. Developmental Psychobiology, 44, 68-83.

Wolfe, C. D., \& Bell, M. A. (2007). Sources of variability in working memory in early childhood: A consideration of age, temperament, language, and brain electrical activity. Cognitive Development, 22, 431-455.

Woodcock, R. W., \& Mather, N. (2000). Woodcock Johnson Psycho-Educational Battery-III. Itasca, IL: Riverside.

Woodcock, R. W., \& Muñoz-Sandoval, A. F. (1996). Batería Woodcock- Muñoz-R: Pruebas de Aprovechamiento. Itasca, IL: Riverside.

Yerys, B., Hepburn, S., Pennington, B., \& Rogers, S. (2007). Executive function in preschoolers with autism: Evidence consistent with a secondary deficit. Journal of Autism and Developmental Disorders, 37, 1068-1079.

Zelazo, P., Craik, F., \& Booth, L. (2004). Executive function across the life span. Acta Psychologica, 115, 167-183.

Zelazo, P., Frye, D., \& Rapus, T. (1996). An age-related dissociation between knowing rules and using them. Cognitive Development, 11, 37-63.

Zimmerman, I., Steiner, V., \& Pond, R. (2002a). Preschool Language Scale, Fourth Edition, Examiner's manual. San Antonio, TX: Psychological Corp.

Zimmerman, I., Steiner, V., \& Pond, R. (2002b). Preschool Language Scale, Fourth Edition, Spanish Edition. San Antonio, TX: Psychological Corp. 


\section{Endnotes}

1 In this paper, ages are written according to standard practice in the field of speech-language pathology. For example, the age $4 ; 8$ corresponds to 4 years, 8 months of age and the age $0 ; 8$ corresponds to 0 years, 8 months of age.

2 The main focus of the longitudinal study involved data collection using a well-established experimental eye-tracking procedure (for a comprehensive review see Fernald, Zangl, Portillo, \& Marchman, 2008). This aspect of the longitudinal study is not further detailed in this paper.

3 This overall pattern of correlations holds for Primarily Spanish Learners and Spanish-English Learners; therefore, all participants are shown together in Table 6. 